University of Redlands

\title{
Morongo Basin Conservation Suitability and Prioritization Model
}

A Major Individual Project submitted in partial satisfaction of the requirements for the degree of Master of Science in Geographic Information Systems

by

Rachel Beard

Fang Ren, Ph.D., Committee Chair

Douglas Flewelling, Ph.D.

December 2009 
Morongo Basin Conservation Suitability Model Copyright (C) 2009

by

Rachel Beard 
The report of Rachel Beard is approved.

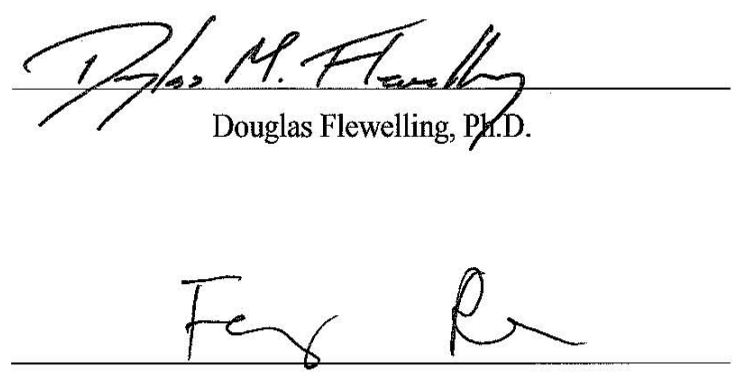

Fang Ren, Ph.D., Committee Chair

December 2009 



\section{Acknowledgements}

I would just like to thank a number of people who helped me develop and complete this project for the MS GIS program. I would like to thank Max Baber, for the issue with my project plan that taught me what project management really is. I am not being sarcastic; I really do appreciate it. I value everything he taught me and the support he gave me when I didn't think I was good enough to be here. I would like to thank Fang Ren for pointing out the issue with my project and providing all the support I needed to address it. I want to thank her for the structure and solid advice she gave me. I would like to thank Doug Flewelling, for forcing me to fix the problem which ultimately resulted in this project's success. Ruben Ortiz, I want to thank for all of the computer support he gave me. Without which neither the computer nor I would have survived this program. Serene Ong, I want to thank for the assistance she provided with some of the technical issues and limitations of ArcGIS. Nathan Strout for ultimately developing the work around to those limitations of ArcGIS and also for helping me make this project easily useable for my client. I want to thank Stephanie Weigel for giving me this awesome project and opportunity to apply GIS to a conservation biology issue. In addition, I want to thank her for giving me the opportunity to meet so many people in the conservation biology and planning field. I know it doesn't seem like much but it informed the direction that I want to take this degree. It introduced me to the complex network of people and challenges that face conservationists across the Western United States. I want to thank Shannon Lear, for going riding with me and convincing me that I really did belong in this program. At a greater extent, I want to thank all of the people involved with Cohort 15. I want to thank them for the friendship and good times that I hope will still exist after we leave this campus. Cheers! 



\section{Abstract \\ Morongo Basin Conservation Suitability Model}

by

Rachel Beard

There exists a variety of detailed data from different sources covering the area within the Morongo Basin being considered for conservation efforts. In addition, a detailed linkage design was developed by conservation scientists with the South Coast Wildlands (SCW) to map the areas that have the greatest potential for wildlife movement and hence make them the prime targets for conservations efforts. The problem was that there was no application that could use the linkage designs and the detailed land information to support in the development of a well informed conservation action plan. The effective advance involved the development of a conservation suitability model that took into account key land information and weighted appropriate features as they would impact the primary purpose of supporting wildlife movement in the area and produced a single suitability score. Features that might impede wildlife movement have been evaluated by the SCW report, however a number of features had not been considered and features that would diminish relative conservability of the land had not been accounted for. The conservation suitability model developed in this project incorporated those features that affected the permeability of the land for wildlife movement as well as features that diminish conservation efforts. 



\section{Table of Contents}

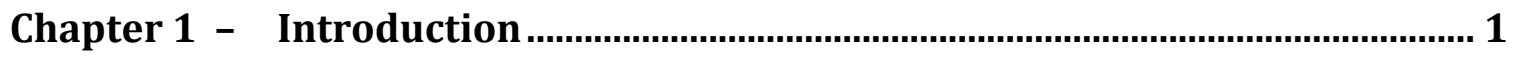

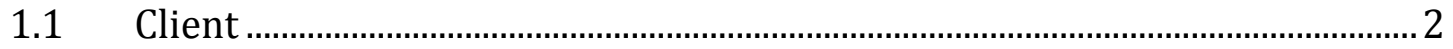

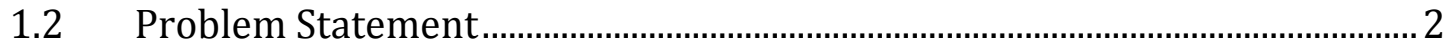

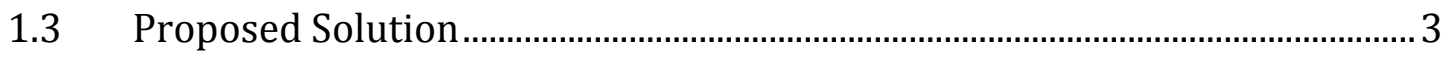

1.3.1 Goals and Objectives …………………………………………………….....

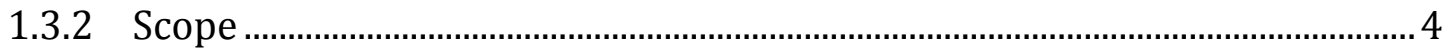

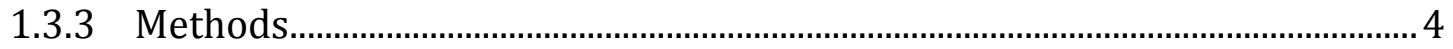

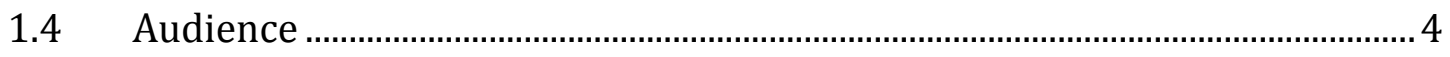

1.5 Overview of the Rest of this Report .................................................................... 4

Chapter 2 - Background and Literature Review................................................... 7

2.1 Wildlife Corridors and the South Coast Missing Linkages Design .................

2.2 Prioritization and Suitability Models................................................................... 8

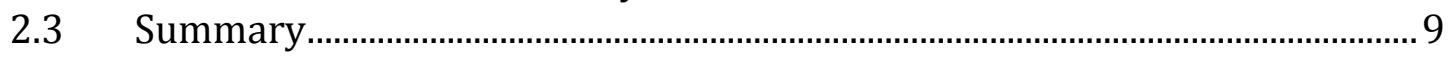

Chapter 3 - Systems Analysis and Design …....................................................11

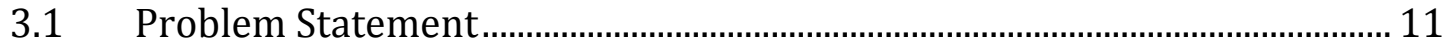

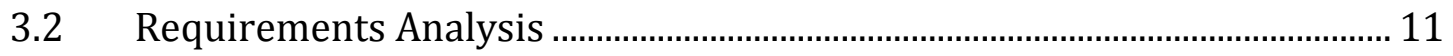

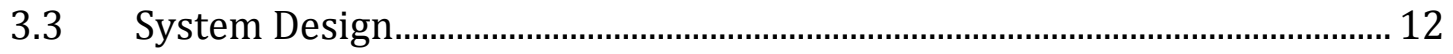

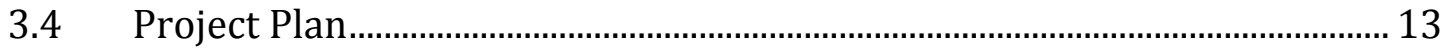

3.4.1 Requirements Analysis ................................................................................ 13

3.4.2 Data Needs Assessment................................................................................... 14

3.4.3 Geodatabase Development............................................................................. 14

3.4.4 ModelBuilder Application/Python Scripting ……………………………….... 14

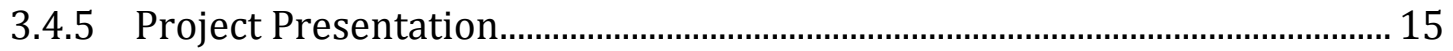

3.4.6 Project Conclusion ....................................................................................... 15

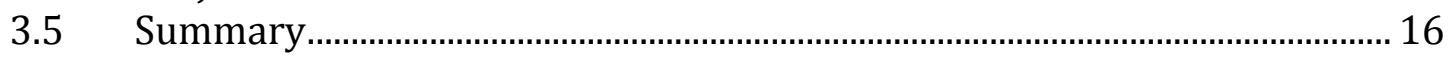

Chapter 4 - Database Design ..........................................................................17

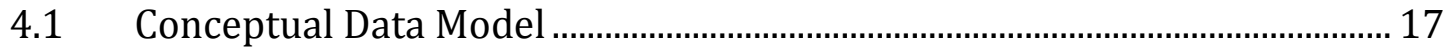

$4.2 \quad$ Logical Data Model........................................................................................... 18

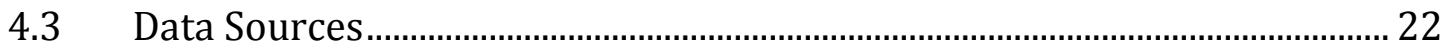

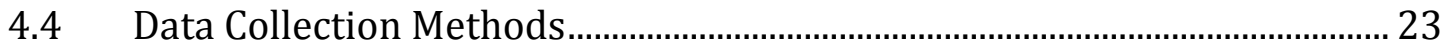

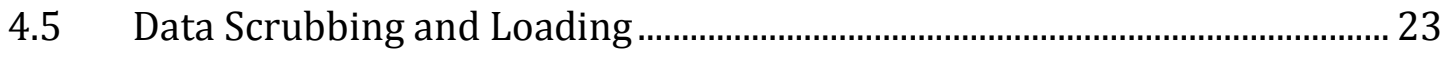

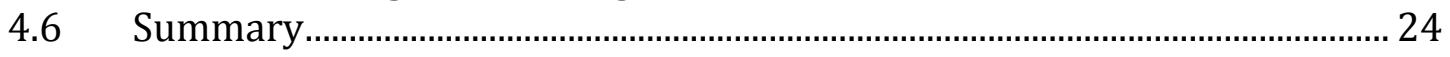

Chapter 5 - Implementation ……..................................................................25

$5.1 \quad$ Criteria Identification and Analysis ................................................................. 25

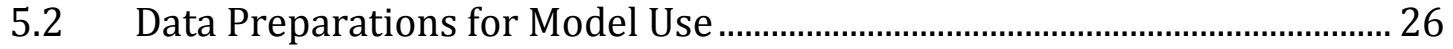

5.3 Model Development and Spatial Analysis........................................................2 29

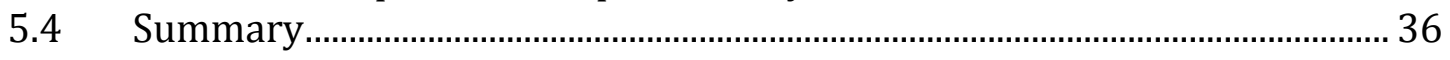

Chapter 6 - Results and Analysis.................................................................37

$6.1 \quad$ Representing the Model Results.................................................................. 37

6.2 Model Results and Adjustments ..................................................................... 39 


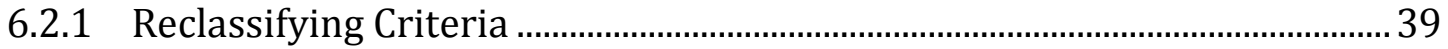

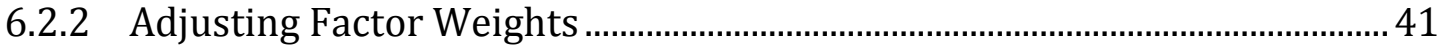

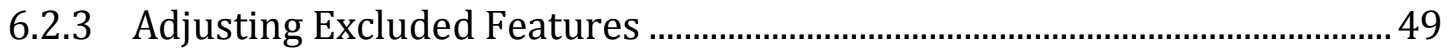

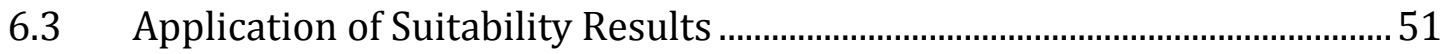

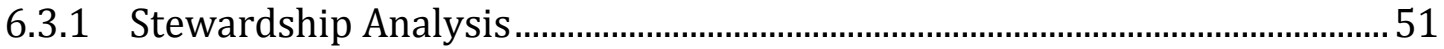

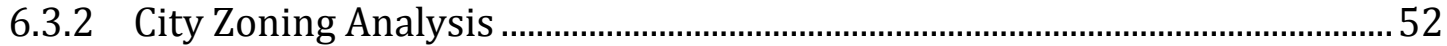

6.3.3 Suitability of Unclassified BLM land................................................................... 54

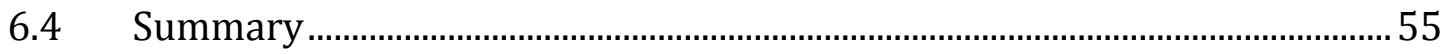

Chapter 7 - Conclusions and Future Work ........................................................57

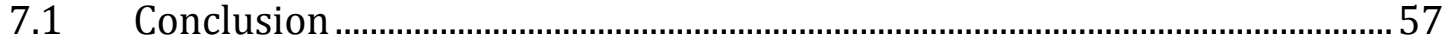

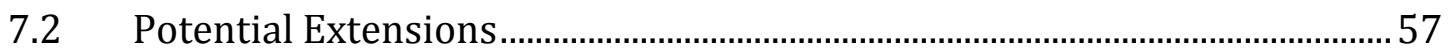

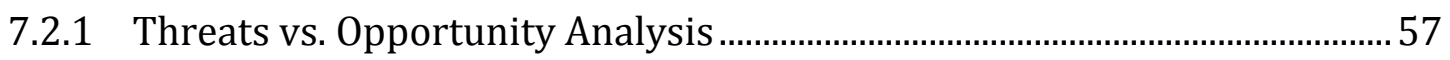

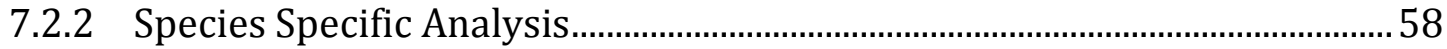

7.2.3 Model Corridor Loss Effects ............................................................................. 58

7.2.4 Develop a General Conservation Prioritization Template and Toolset..... 58

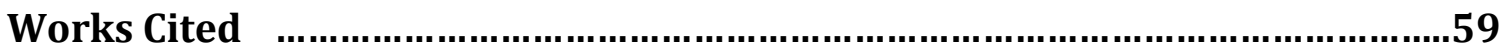

Appendix A. Density Line Density Calculation Python Script............................61

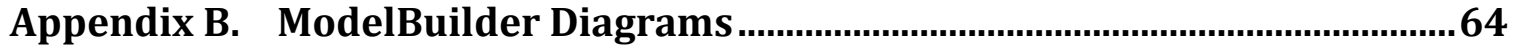

Appendix C. Suitability Score Calculation Python Script ...................................69 


\section{Table of Figures}

Figure 1-1: Morongo Basin Open Space Group Focus Area and Linkage Design......2

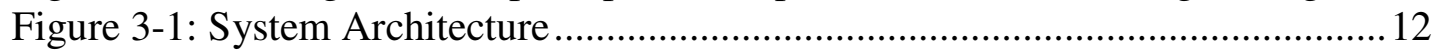

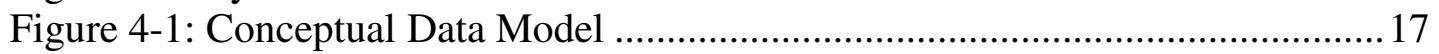

Figure 4-2: ESRI Diagrammer Image of the Database Design............................... 18

Figure 4-3: Feature Datasets and Feature Classes ...............................................20

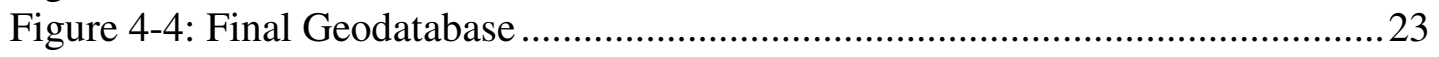

Figure 5-1: Density Calculation Error Message ................................................28

Figure 5-2: Criteria Classification Geodatabase Table ......................................... 31

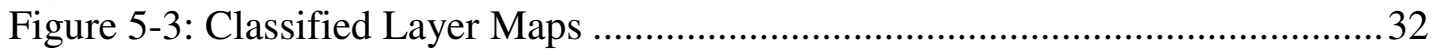

Figure 5-4: ModelBuilder Addition of a Reclass Field ......................................... 33

Figure 5-5: ModelBuilder Criteria Weighting and Scoring .................................... 34

Figure 5-6: ModelBuilder Final Suitability Score Calculation ................................35

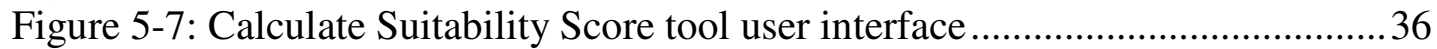

Figure 6-1: Initial Parameters ......................................................................... 37

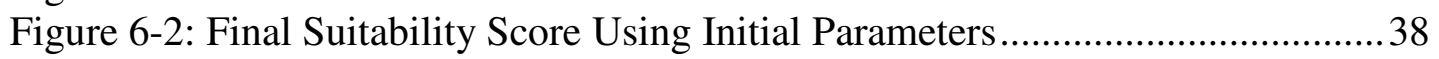

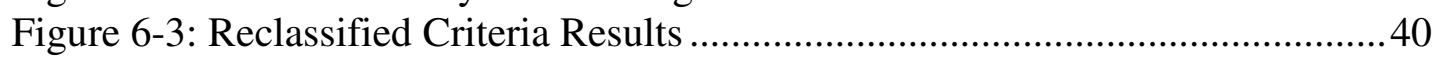

Figure 6-4: Linkage Design Weight Adjustment.................................................. 43

Figure 6-5: Conservation Suitability Weight Adjustment .....................................46

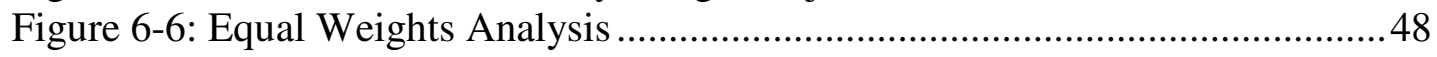

Figure 6-7: Exclusive Linkage Design Suitability Analysis ...................................50

Figure 6-8: Exclusive Linkage Design Suitability Analysis....................................52

Figure 6-9: Rural Living and Suitability Score Analysis.........................................53

Figure 6-10: Undesignated BLM Land Suitability Analysis ..................................54 



\section{List of Tables}

Table 1. Summary of Requirements ................................................................ 11

Table 2. Morongo Basin Conservation Suitability Model MIDL ............................. 22

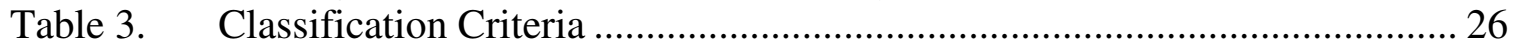

Table 4. Weight Calculation Spreadsheet in Excel................................................ 30

Table 5. Linkage Design Weights Adjustments Table ........................................... 42

Table 6. Opportunity Weight Adjustment Spreadsheet ............................................ 44

Table 7. Equal Weight Adjustment Spreadsheet ................................................ 47 



\section{List of Acronyms and Definitions}

$\begin{array}{ll}\text { APN } & \text { Assessors Parcel Number } \\ \text { BLM } & \text { Bureau of Land Management } \\ \text { DOD } & \text { Department of Defense } \\ \text { DVD } & \text { Digital Video Disc } \\ \text { FEMA } & \text { Federal Emergency Management Agency } \\ \text { GIS } & \text { Geographic Information System } \\ \text { MBOSG } & \text { Morongo Basin Open Space Group } \\ \text { MCAGCC } & \text { Marine Corps Air Ground Combat Center } \\ \text { NPS } & \text { National Park Service } \\ \text { OHV } & \text { Off Highway Vehicle } \\ \text { SCW } & \text { South Coast Wildlands } \\ \text { TWC } & \text { The Wildlands Conservancy }\end{array}$





\section{Chapter 1 - Introduction}

This chapter further introduces the client, the client's problem and needs, and how the model was designed to address those needs. In addition this chapter explains the tone and direction of the remaining chapters. The Morongo Basin conservation prioritization model was designed to meet the needs of Stephanie Weigel and the Morongo Basin Open Space Group (MBOSG). Weigel and the MBOSG wanted a tool that would assist in the visualization of the conservability of land that interacts with the linkage design developed by South Coast Wildlands (SCW).

SCW is a not-for-profit group that works with ecologists, biologists, land managers, and planners, as well as conservation organizations, to develop conservation strategies. Their mission is to protect and restore ecosystems that support wildlife and promote their movement between functional habitats. They have worked in collaboration with many groups similar to the MBOSG to connect over 18 million acres of existing conservation

areas in the South Coast Ecoregion and are currently extending their efforts to the Mojave Desert with projects such as the Joshua Tree National Park-Twentynine Palms Linkage. This linkage design has prioritized areas within the Morongo Basin as the most suitable for wildlife movement. The MBOSG wants to conserve the areas that interact with these linkages so that movement can be maintained in perpetuity.

The linkage design created for the Joshua Tree-Twentynine Palms area was targeted because this area supports a great diversity of species. Joshua Tree National Park has been reported by the National Park Service (NPS) to support more than 1049 plant and animal species and the Twentynine Palms Marine Corps Air Ground Combat Center (MCAGCC) has reported to support more than 650 vertebrate and vascular plant species (Figure 1-1). Many conservation investments already exist in the area because of the efforts taken by The Wildlands Conservancy (TWC), The Bureau of Land Management (BLM), and State Lands Commission. However, there are holes in the currently conserved land that must in some way be protected to ensure wildlife movement in the area. For this reason, the MBOSG and the stakeholders involved in it have made it their goal to protect and promote the movement of these species between the two targeted areas. 


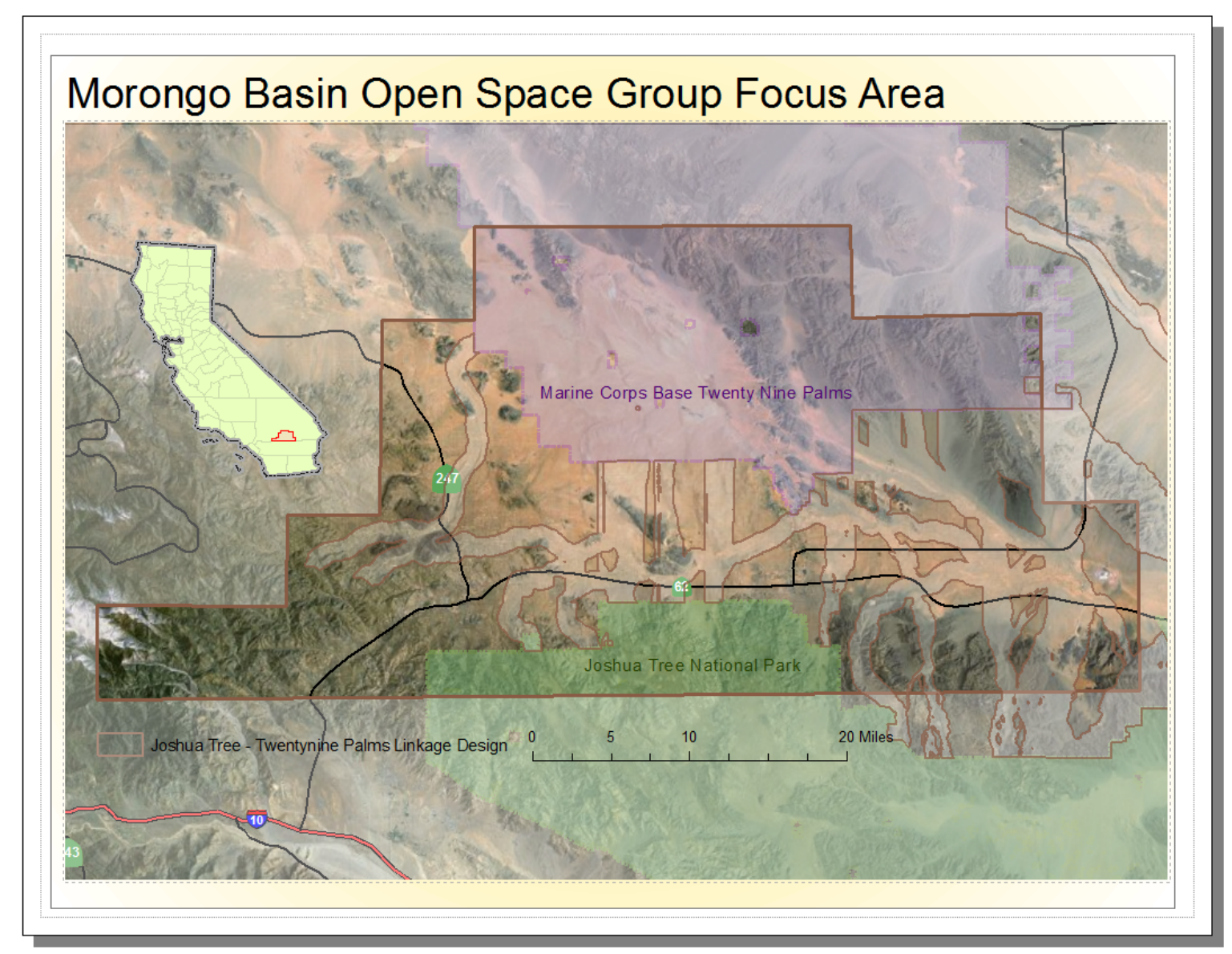

Figure 1-1: Morongo Basin Open Space Group Focus Area and Linkage Design

\subsection{Client}

Stephanie Weigel is the regional land use planner working with the Morongo Basin Open Space Group (MBOSG). The MBOSG is a collaborative multiagency and community group that plans for the future development and conservation of the Morongo Basin. Their goal is to collectively identify the common interests regarding open space, conservation, preservation, and stewardship opportunities. The MBOSG encourages the participation of the community in the planning process by attendance at the MBOSG bimonthly meetings. One of the major goals of the MBOSG is to prioritize the land in the basin for conservation and identify areas to focus stewardship efforts. To help these efforts, the South Coast Wildlands (SCW) has done an extensive analysis of species movement potential in between Joshua Tree National Park and Twentynine Palms Marine Base and produced a linkage design.

\subsection{Problem Statement}

It is unreasonable to assume that all of the available areas that interact with the linkage design can be conserved. The MBOSG needs to prioritize these areas and develop a well informed conservation action plan. Weigel had compiled a variety of digital data 
covering the MBOSG focus area that she used for basic analysis and local spatial information dissemination. She used these data to help the stakeholders visualize the complex spatial interactions that take place in the basin and how they relate to the goals of the MBOSG. The problem was that she did not have an efficient way to evaluate the basin quantitatively and compare one area against another. The group had originally been prioritizing areas in the basin through meeting and workshop sessions. These meetings and workshops could be enhanced by the availability of a tool that could quantitatively help stakeholders quickly visualize and understand the complex interactions that go into the conservability of the land.

In addition, the MBOSG wanted to understand the features in the landscape that would conflict with the ultimate goal of conserving the land for wildlife movement. Several features that might impede wildlife movement had been evaluated by the SCW. However, a number of features that would diminish or enhance the relative conservability of the land had not been accounted for. Some of these features include BLM-designated OHV (Off Highway Vehicle) Routes and land ownership or management. These factors needed to be evaluated, along with the factors that affect wildlife movement.

\subsection{Proposed Solution}

The designed solution was to use Geographic Information System (GIS) to develop suitability model that takes into account identified key land information and weights appropriate features according to their impact on potential wildlife movement and the conservability of the land. With this tool, the client is able to assess the relative suitability of individual parcels for conservation. More importantly, the tool should be repeatable in the future to accommodate the dynamics in the region, such as the changing land ownership and land cover, and should be modified to adapt to the potential changes in the client's goal. The designed suitability model was implemented in ArcGIS platform using weighed overlay technique. Several custom models that serve the overall suitability model were developed with ArcGIS ModelBuilder and several custom functions were programmed using Python scripting.

\subsubsection{Goals and Objectives}

The ultimate goal of this project was to develop an easy-to-use tool for MBOSG to visualize the land conservability with the linkage design developed by South Coast Wildlands. More specifically, the project aimed to develop a GIS model to assess the suitability of each parcel in the basin for conservation based on a set of predetermined criteria. The model output was designed to meet the needs and conditions of the MBOSG and be able to be used for the development of reports and informative maps. This model was designed to be easy to use by a user who has moderate experience in ArcGIS software. The model was also designed to be repeatable and modifiable to adapt to the characteristics in the basin and the goals of the MBOSG. 


\subsubsection{Scope}

The model was designed to easily assess the suitability of individual parcels within the MBOSG focus. The MBOSG focus area is defined by the Morongo Unified School District boundary. The focus area covers $\sim 1600$ square miles and contains $\sim 69,000$ parcels. Because the assessment was parcel-based all of the criteria datasets had to be processed to be attributes of the parcels with an APN assigned to each. There were nine criteria that had to be processed in order for the suitability assessment. This preprocessing was not automated for the client because of time constraints.

The final product of the project was a conservation suitability tool that would produce a final score between 1 and 5. This model is designed to be modifiable and repeatable. All of the criteria classification ranges and weights can be easily adjusted to fit the needs of the end user. It was beyond the scope of this project, however, to automate that attribute preparation and the addition and removal of criteria from the model. These processes however have been documented and can be performed by an experienced GIS user.

\subsubsection{Methods}

It was determined that this project was a variation of a land suitability analysis that involved multiple criteria to assess the landscape for conservability. This project used GIS-weighted overlay techniques, ModelBuilder, and custom scripting in order to identify areas of greatest suitability. The weighted overlay process had to be completed within a ModelBuilder environment because this project focused on scoring the parcels themselves rather than producing a suitability surface. In order to complete a weighted overlay analysis with vector data the criteria data had to be processed to be attributes of the individual parcels. Once these processes were completed the criteria then had to be classed according to their impact on the suitability of that parcel for conservation. ModelBuilder was then used to automate the weighting and calculation of the final suitability score. In order to complete this automation process Python scripting was used to join a table in which the user could adjust all the parameters to the calculation model. With the use of Python scripting the end user will only interact with a basic tool interface and the suitability classification table.

\subsection{Audience}

The intended audience for this paper is primarily Stephanie Weigel, the regional land use planner for the Morongo Basin, in addition to land managers as a whole. Because these individuals have varying levels of GIS knowledge, the overall tone of this paper will be addressed to an audience that is unfamiliar with GIS processes but understands its application.

\subsection{Overview of the Rest of this Report}

The paper has been structured to follow the development processes that were used to complete the project. Chapter 2, the literature review, will discuss the research and 
projects that informed the development of this project. Chapter 3 will discuss the system analysis and design. The system analysis enumerates the process that went into the design and plan of the project development. Chapter 4 describes the decisions that were made when developing the geodatabase that would support the model. Chapter 5 explains in detail the methods that were used in the model's development and implementation. Chapter 6 discusses the results and the analysis of the project. It discusses the results of the suitability model and the potential uses of those results. Finally, Chapter 7 is reflection of the whole project process; the successes, challenges and failures. It also explains how the project met the requirements of the client and discusses the need for future extensions of this project. 


\section{Chapter 2 - Background and Literature Review}

The South Coast Wildlands developed a linkage design for the Morongo Basin. The MBOSG's primary goal is to develop an action plan to conserve or set development and stewardship standards in areas that interact with the linkage design, and to prioritize their efforts to meet this goal. The research described in this chapter looks into the value and importance of wildlife corridors and the theories that informed the development of the Joshua Tree-Twentynine Palms linkage design. This research review was conducted to better understand the theories behind wildlife corridors and the criteria that affect wildlife movement. In addition, this chapter examines the ways that GIS has been used in suitability analyses by past researchers and organizations. All these studies shed light on the methods suitable for this project.

\subsection{Wildlife Corridors and the South Coast Missing Linkages Design}

Many biologists and conservationists have researched the effects of habitat fragmentation and urbanization on species movement and population survival, as well as the effectiveness of wildlife corridors (Mader, 1984; Haddad, 1999; Schmiegelow et al., 1997; Wolff et al., 1997; Debinski, 2000). Although these studies were not conducted for the arid Mojave Desert they provide valuable empirical evidence and shed light on the methodology that could be applied to this project.

For example, twenty studies had been done worldwide by 1998 to explain the complexity of habitat connectivity and the challenges of mitigating the effects of human presence (Debinski, 2000). Habitat fragmentation causes limitations to dispersal of species which results in altered behavior and diminished species richness in the area (Mader, 1984). Research studies suggest that corridors mitigate the effects of fragmentation. Several studies (Schmiegelow et al., 1997; Wolff et al., 1997; Haddad, 1999) have shown that corridors increase the movement and abundance of species. Haddad and Baum (1999) found that corridor areas had higher densities of the focal butterfly species compared to insular habitats. This research provided the foundation that the South Coast Wildlands (2008) used to develop their corridor plans.

An increasing number of studies have been done since then, some of which use GIS as an aid. They have a range of focus, many having to do with species richness or abundance, with a few exceptions focusing on wildlife corridors and landscape connectivity (Debinski, 2000).

Among the various causes for habitat fragmentation, transportation networks create a significant hazard to wildlife species and connectivity. In this plan the effects of urbanization and transportation networks on connectivity are considered. Roads do more than just kill animals; they actually act as an impermeable boundary for many small animals and a semipermeable one for larger animals (Forman and Deblinger, 2000). The result of these effects is a stagnant genetic flow that increases the risk of extinction 
(Gilpin and Soulé, 1986). Forman and Deblinger (2000) suggest several ways to manage and mitigate the effects of road barriers. The most prominent suggestion is to create wildlife crossing structures such as overpasses and underpasses, which meet the needs of various focal species.

Urban development poses the second largest threat to habitat connectivity and is more difficult to mitigate. Both urban and suburban areas limit the movement of species by removing habitat, creating edge effects, and by introducing wildlife hazards such as predatory pets and poisons (Hall et al., 2000, Penrod et al., 2008). In the Joshua TreeTwentynine Palms linkage design, Penrod et al. (2008) discussed how outskirt residential areas are the only urban features that threaten the permeability in the area. To mitigate these potential regions of impermeability, Marzluff and Ewing (2001) suggested creating zones of stewardship. Residents in these areas would be encouraged to enforce lower speed limits, limit irrigation, minimize the use of herbicides and pesticides, and encourage the use of native plant species as opposed to exotics in landscaping.

Different approaches were applied among the past studies, among which GIS approved to be very useful. For example, several studies have used GIS to better understand how major transportation networks affect the movement of wildlife (Treweek and Veitch, 1996; Smith, 1999). Modeling habitat linkages with a GIS have been used to determine the placement of wildlife crossing structures as well as predict the consequence of unmitigated barrier features. For example, Clevenger et al. (2002) used a GIS to model the success of wildlife corridors across a Canadian transportation network. Similarly, Theoblad (2002) designed a GIS model that examined the consequences of land changes, such as the development of roads, and the fragmenting effects. This type of predictive modeling addresses an important question: is the development of this feature going to increase fragmentation?

South Coast Wildlands embraced the theories obtained from these empirical studies in their creation of A Linkage Design for the Joshua Tree-Twentynine Palms Connection in which they specified suggestions to mitigate the effects of roads on focal species (Penrod et al., 2008). They suggested creating crossing structures and improving existing underpasses, as well as reducing speed limits, and creating small culverts to accommodate tortoises and other small animal species. In addition, Penrod et al (2008) and the South Coast Wildlands (2008) created the framework for local coalitions to make informed decisions about conservation measures and realize the barriers and conflicts that exist that limit the effectiveness of the corridors.

\subsection{Prioritization and Suitability Models}

Many researchers and organizations have used GIS to analyze the land's suitability for a project or to prioritize the land for action. Land suitability analysis is defined by Steiner and McSherry (2000) as the process of determining the fitness of an area for a given use, as well as to determine its suitability level. In this process, spatial characteristics of the area and how they interact with a proposed use should be taken into account. Among various factors that are used for land assessment, three factors seem to be always relevant 
and important: location, development, and environmental processes (Al-Shalabi, 2006). GIS has proven to be an effective tool to evaluate these factors simultaneously and has been suggested as a decision support tool (Jankowski, 1995).

The goal of land suitability analysis is not to isolate areas that have the best potential, but to map and index the suitability of entire focus areas. Map overlays and classification techniques were used in many past land suitability analyses ((Hall et al., (1992); Wang, (1994) Joerin et al., (2001)). Using multi-criteria techniques and GIS modeling, researchers and organizations have developed decision support models for site selection and prioritization.

Al-Shalabi et al. (2006) used GIS and multi-criteria ranking techniques to identify suitable areas for housing development. This decision support system was based on a set criteria derived from the spatial aspects of the environment, local and national policies, and the local physical plan. These criteria were then evaluated based on development preference factors and given weightings based on importance. The model they used was a set of spatial processes such as buffer, classification, reclassification, overlay and weighted overlay techniques. They describe this process as commonly used in site suitability analysis where multiple factors have an effect on the suitability.

Similarly Schultz et al. (2003) developed a land suitability model using the weighted overlay technique to identify land that was most suitable for development and conservation within several Mississippi counties. In their weighted overlay modeling process, the considered variables were grouped into four categories: Government Owned land/Restricted Development, Land Use/Land Cover, Hydric Soils, and Federal Emergency Management Agency (FEMA) Flood Zones. The land areas available for potential development were assigned a scale of one to nine, one being lowest level of suitability and nine being the highest. The model calculates the outcome score for each cell and its relative influence. The result is a map showing each cell's total score. This suitability model and its resulting maps are used to inform comprehensive planning efforts in those Mississippi counties.

Similar to suitability analysis and decision support models, the Pacific Biodiversity Institute (2002) developed a prioritization model and conservation decision support system that is used to inform and guide organizations in their conservation efforts. This model used a variety of inputs and provided the contracting organization with an easy-touse interface even for non-GIS users. In addition, a user could overlay the parcel information and interpret the average conservation value from prioritization. This process allows for the ranking of private lands.

\subsection{Summary}

In this chapter the value and importance of wildlife corridors was researched. Possible impediments to wildlife movement and suggested ways to mitigate those impediments were also identified from the literature. Drawing upon the findings from past studies, South Coast Wildlands has evaluated the Joshua Tree and Twentynine Palms area and 
developed a linkage design to identify the least cost areas for wildlife movement. Although this linkage design identifies areas of greatest likelihood for wildlife movement, MBOSG still needs to develop a plan to conserve these areas as the group cannot conserve all of the land within the linkage design. Therefore, a land suitability analysis was conducted in this project to help the group assess the land for conservation suitability and prioritization.

Many researchers and organizations have used suitability assessment and prioritization models to assess land in a given focus area. These models take into account the many factors that go into the decision process and use GIS to assess areas that best or least suit the desired action. Past studies have suggested the process for suitability assessment and prioritization, which includes determining focus criteria, setting criteria weights and importance, and assessing these in a weighted overlay process. The project designed for the Morongo Basin similarly used these processes. 


\section{Chapter 3 - Systems Analysis and Design}

This chapter will discuss the key topics of this project regarding to system design and analysis: the problem that was addressed by this project; the analysis that went into deciding the project requirements and data needs; the system design of the project; the project plan; and a general overview of the steps taken in order to complete the project.

\subsection{Problem Statement}

The MBOSG is an organization that collaborates with local agencies and organizations to protect the diversity in the Morongo Basin through conservation, preservation, stewardship, and regional land use planning. Currently, MBOSG in making their decisions to use the linkage design developed by the SCW as a template in its conservation efforts. Since it is unreasonable to assume that all of the land in the linkage design can be conserved, it is necessary to prioritize the parcels.

In the process of the prioritization of parcels, the group has to know the basic parcel data, such as ownership size and location, as well as how appropriate it is for conservation. The determined criteria for conservation suitability were primarily land cover attributes and potential connectivity with other conservation lands. Therefore, the problem that MBOSG faced was how to evaluate the land suitability for conservation by taking all these factors into account.

\subsection{Requirements Analysis}

The client wanted an application that could be easily used by a knowledgeable Arc GIS user to develop informative maps and reports. The application was designed to be modifiable and repeatable so that the user can update the data and the model as needed, as well as apply the model to future project areas.

The primary functional and non functional requirements for this project were specified by the client and incorporated into the application. Table 1 provides a summary of those requirements.

Table 1. Summary of Requirements

\begin{tabular}{|l|l|}
\hline Nonfunctional (What) & Functional (How) \\
\hline \hline Ease of use & ModelBuilder to automate workflow \\
\hline Light data storage & File geodatabase storage \\
\hline Updateable & Use updated data sources \\
\hline Modifiable & Use existing regional data \\
\hline User documentation & Instruction manual \\
\hline
\end{tabular}


The primary nonfunctional requirements were that the model be updateable, modifiable and repeatable. The model had to be updateable because information in the Morongo Basin is always changing. New developments may be approved in the basin and new areas are being conserved or transitioned into military base or National Park lands. It is also possible that the client will need to evaluate land suitability in other areas in the future projects. To accommodate the updated information or new study areas, the land evaluation procedure should be automated and simple such that it is easy to repeat it. Therefore, an automated GIS model was required to realize these nonfunctional requirements. In addition, a geodatabase was necessary for data storage and organization to assist in the model development as well as data maintenance.

\subsection{System Design}

The client's functional and non functional requirements dictated the system design which includes system requirements, operating environment, geodatabase design, and interfaces. This application was designed to be accessed from a computer with ArcGIS 9.3 or higher with a geodatabase configured for data storage and organization. These components, the model and the database, will be described here as the primary elements of the system design (Figure 3-1).
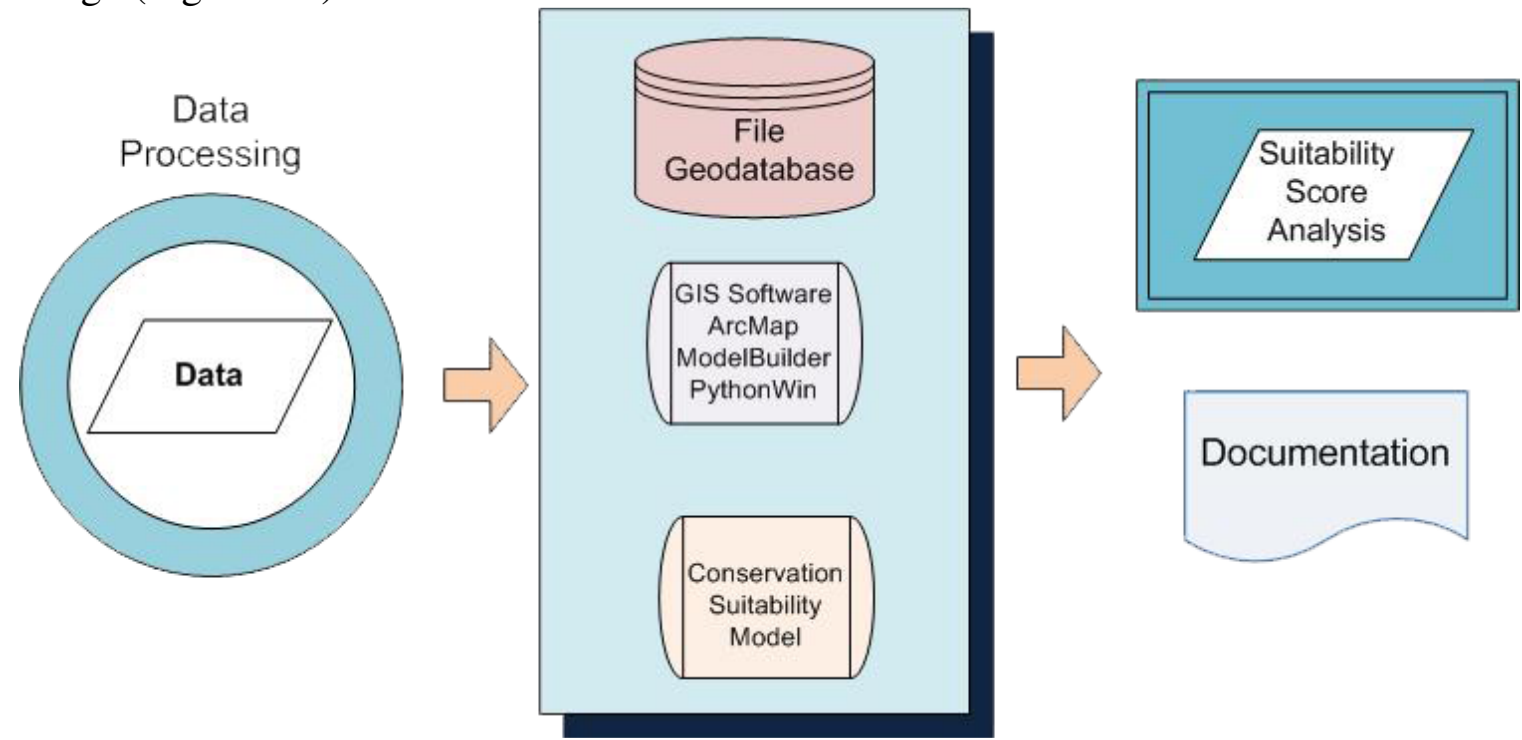

Figure 3-1: System Architecture

The system requirements and operating environment for this project are minimal. This model can be used on individual workstations that are compatible with ArcGIS programs that have an ArcInfo license. In addition, Python Win needs to be installed to run the Python scripts that are necessary for the model. Python Win is available with ArcGIS at all licensing levels.

The data that were required to meet the goals of the MBOSG are: basic parcel data, land use data, linkage design components, Department of Defense expansion plans, currently conserved lands, and public land data. However, within the geodatabase the 
data were divided into administrative units, conservation, transportation, and land cover. These broad descriptions will allow the client to insert other datasets that may be useful in future work. These data were processed to be attributes within the parcel data. This process was well documented and partially automated to assist the client in future data preparation processes.

The model development was done solely with the ArcGIS Modelbuilder and was supplemented by Python scripting for those custom functions. Python is a general purpose object oriented programming language that works well with many programs. Python scripting was used as opposed to other languages because of its relative ease of use, readability, and compatibility with ArcGIS. The output of the model produces a result in the attributes of the parcel data. This can be mapped for visual graphics or used to produce a report.

\subsection{Project Plan}

Many similar land use suitability applications have been developed to address similar needs and requirements. This project is a variation of those suitability analyses and the processes of those projects were adopted in this project plan. The primary tasks for this project were:

- Requirements analysis

- Data Needs assessment

- Database design

- Build ModelBuilder applications/Python scripting

- Project documentation

- Public presentation

- Project conclusion

These tasks were generally completed in chronological order using the waterfall model. The processes were done sequentially. The exception to this was the documentation process which was done concurrently with all of the other tasks and written as it applied to the completed processes.

\subsubsection{Requirements Analysis}

The requirements analysis evaluated the needs and capabilities of the client. This task required several discussions with the client to determine data capabilities, software and hardware, and to survey the available datasets. The client had all of the appropriate technical requirements for a desktop ArcGIS application and was able to supply all of the 
needed datasets and data storage capabilities. The client participated in the defining the critical data requirements, developing the system requirements, and provided insight and feedback during the modeling process.

\subsubsection{Data Needs Assessment}

The client provided all of the datasets needed for the project. The active datasets needed for this project were: parcel data, hydrography data, roads, BLM Off Highway Vehicle (OHV) designated areas, vegetation, protected areas, SCW linkage design, and Department of Defense (DOD) base expansion plans. Many of these datasets had adequate documentation and were satisfactory to meet the goals of this project. Two datasets had no documentation and their lineage had to be traced and they had to be replaced. The client identified several datasets that would have been valuable in this assessment but did not exist for this area, including: fence lines, gutters and curbs, and building footprints.

\subsubsection{Geodatabase Development}

After the requirements analysis was complete and the data needs were specified, the database design and loading could be completed. It was decided that a file geodatabase would be used both because file geodatabases are recommended for use by ESRI and because it was the common data storage format used by the client and the SCW project. The database was designed following the organizational structure of the SCW database. The SCW database schema was used in the preparation of the linkage design and had a simple structure so it could be easily modified and updated to fit other projects in the future.

The key difference between the SCW project's database and this was the emphasis on parcel data. This project was intended to prioritize parcels based on their suitability for conservation. For this reason the parcel data had to be the central focus of the database as well as the model processes.

\subsubsection{ModelBuilder Application/Python Scripting}

Many of the past projects in the literature documented the model building process for land use suitability analysis, which use either the raster or vector data models. For this particular project it was originally decided that the analysis would be performed within the raster data model. The raster data model represents features as a matrix of cells across continuous space. Each layer can represent only one attribute and analysis can be performed by combining the layers into to a new one with new cell values. Many of these processes can be performed with the tools available with ArcGIS Spatial Analyst and require little to no customization. The advantage of using a raster data model is that multiple raster layers can be evaluated at once with relatively little computer resources. If these processes are performed in vector format, much more computer resources and customized tools are required. 
To complete this project using the raster data model, all of the data would have to be converted to raster datasets and evaluated within Spatial Analyst. Converting all of the parcels and associated data into raster data would create a inflexible model where only the weights of the individual datasets could be adjusted by the user. All of the other parameters would be determined by the developer in the data preparation process, such the raster resolution at which the vector data were converted. In order for the client to change those parameters, the raster data would have to be recreated with the new parameters. This approach would have resulted in a model that ran quicker but it would have diminished the modifiability significantly.

It was then decided that the model be created by maintaining the vector data format and customizing tools that could perform a weighted overlay. This decision greatly increased the amount of work that was involved in the development process and reduced the time available for interface customization. This decision also increased the functionality of the project and the client's control on the input parameters of the model. In addition, the conversion process of vector to raster has many technical issues that cause information to be lost. When keeping the data in its original data model, this data loss is avoided.

The intermediate steps in the model development remained unchanged by the decision to process the data in vector data model rather than raster, including the criteria determination, classification, and weighting. The final GIS overlay process to combine factors and constraints with the weighting overlay process in ModelBuilder fundamentally remained the same but the creation process was dramatically different.

\subsubsection{Project Presentation}

A presentation was prepared and presented to the MBOSG. The purpose of this presentation was to inform the stakeholders in the MBOSG of the project and results and what it could be used for in the future. It also provided a forum for a question and answer session and to address potential future projects. This presentation included a Microsoft Power Point, handouts, and map posters.

The Power Point slides provided a visual aid to the processes and purpose of the project and the results that were obtained. The handouts provided background information that could not be covered in the time given for the presentation. Handouts provided context and introduce the topics of discussion. Finally the map posters were displayed at the meeting so that stakeholders could easily view and interpret the results of the analysis.

\subsubsection{Project Conclusion}

The project was concluded with the delivery of the project Digital Versatile Disc (DVD) and project documentation to the client. The project DVD contained the final approved report, the file geodatabase, metadata, the model builder applications, and the technical documentation. The project documentation included the detailed project report, the file 
geodatabase, associated datasets, model applications, and the prepared technical documentations.

\subsection{Summary}

The system design was dictated by the client's specified needs and goals, as well as the technical capabilities of the work environment. With these needs and capabilities clearly defined, it was possible for the project to go forward in the development process beginning with geodatabase development and model building process. The decision to change the data model used in the project caused several changes in the project development and planning process but resulted in a efficient model that better suited the need of the client. 


\section{Chapter 4 - Database Design}

\subsection{Conceptual Data Model}

The design of the conceptual data model was based on similar projects that were researched and described in Chapter 2. The client identified the key attributes and datasets to be analyzed in the prioritization model. The goal of the GIS model was to rank parcels within the MBOSG focus area for conservation. For this reason the parcel dataset is in central to the data model. The other associated datasets can be reduced down to characteristics of these parcels. Figure 4-1 illustrates the relationship of some of the considered datasets and their ability to be drawn back as attributes of the parcels dataset.

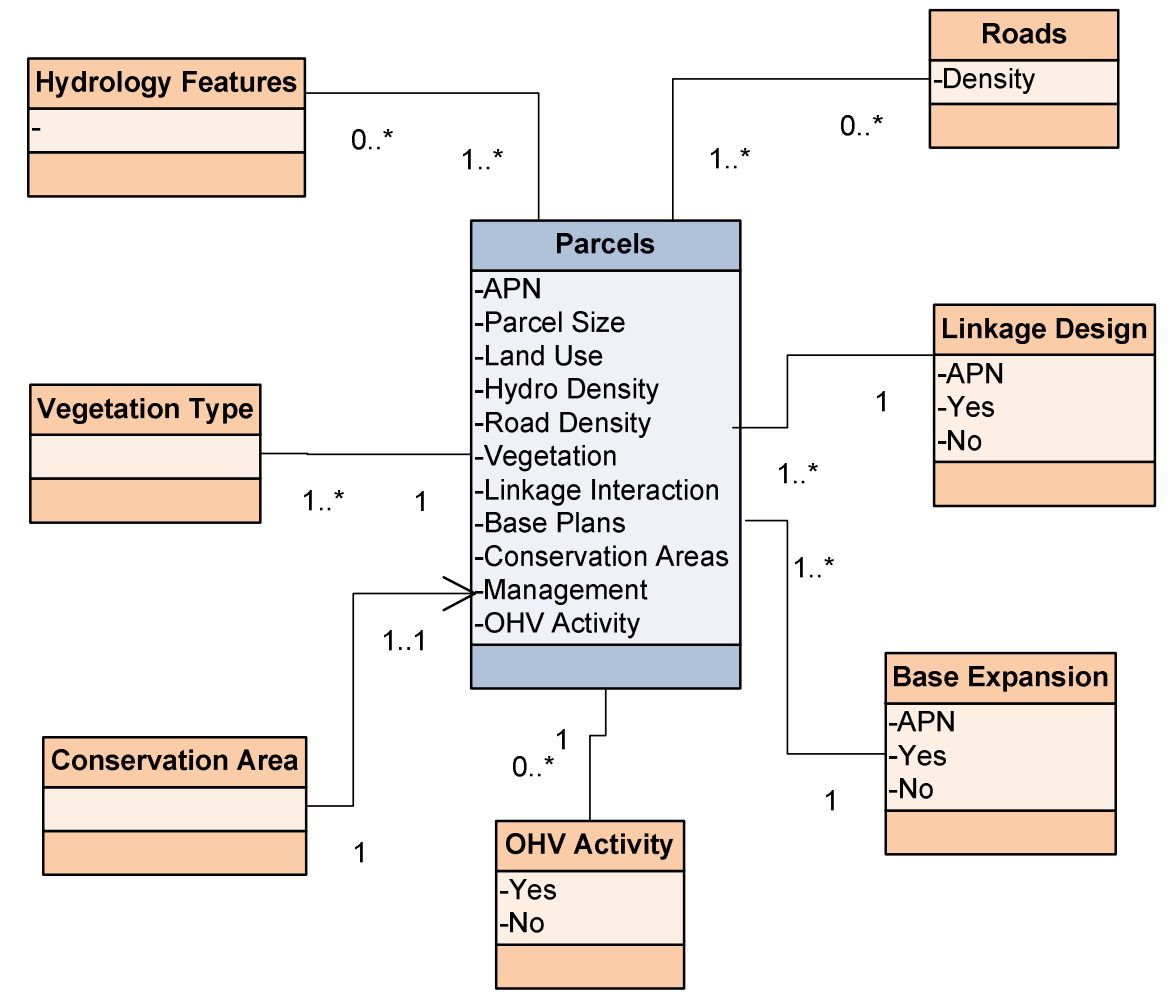

\section{Figure 4-1: Conceptual Data Model}

This figure demonstrates that each of these features can be applied as attributes within the parcel data set. For example, Off Highway routes activity was not included in the original parcel dataset. Using geoprocessing each of these datasets can be transformed to be attributes within the parcel data. It is however important to retain the datasets in the geodatabase. The geodatabase was not meant to be comprised of only the extended parcel dataset. 


\subsection{Logical Data Model}

The primary decisions that were made in the development of the logical data model were what geodatabase type would be used, how the geodatabase would be structured, and what spatial data model would be used in the analysis.

The geodatabase type that was decided upon was the file geodatabase. The file geodatabase was chosen because of its increased size capacity and increase data stability. This geodatabase was structured simply for organizational purposes. The datasets were grouped in to feature datasets that were generally descriptive of the commonalities among the datasets. The parcels and zoning datasets were grouped together for example (Figure 4-2).

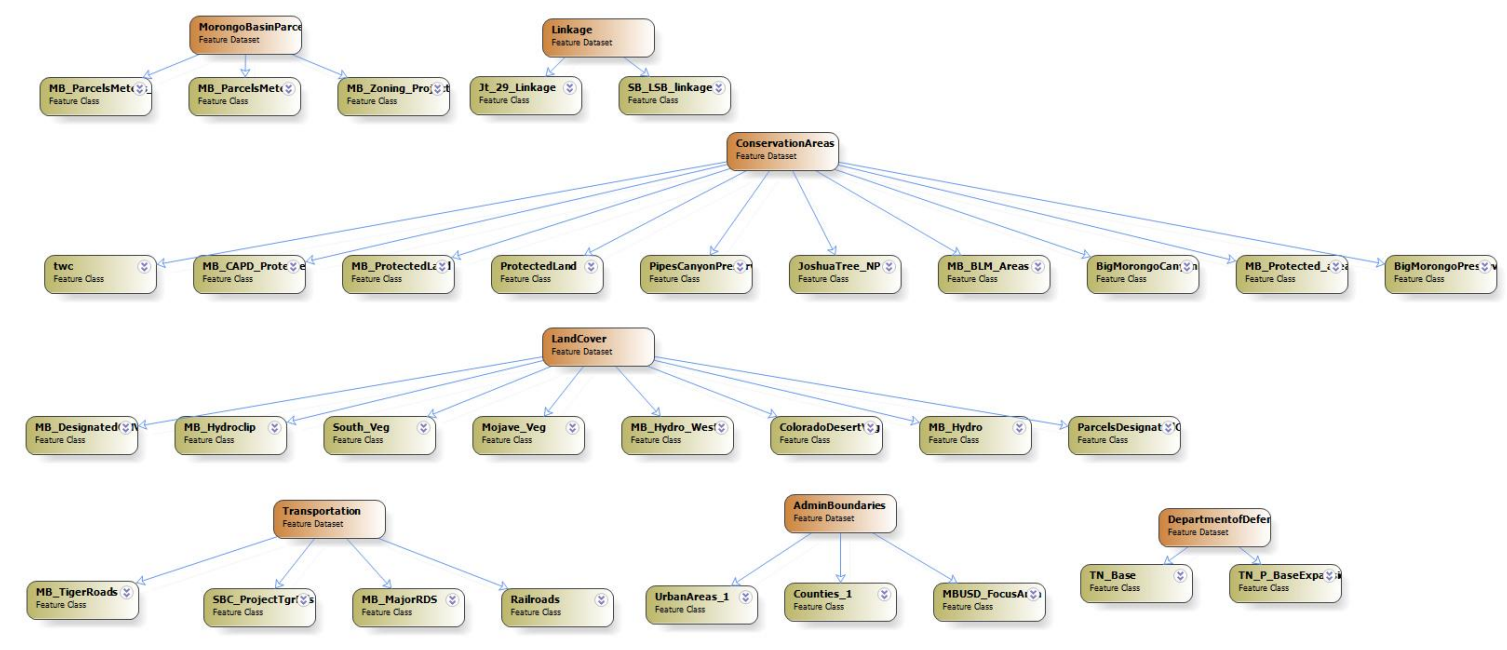

\section{Figure 4-2: ESRI Diagrammer Image of the Database Design}

The seven feature datasets contains the focus datasets used in the creation of the project parcel dataset. These seven feature datasets were designed to provide organization for the data classes contained within them. Some of these datasets were clipped to the focus area these datasets were named with a MB prefix to indicate that they were clipped to the Morongo Basin. All of the datasets that were used in the creation of the project parcel data set and were retained because they contain the valuable dataset specific information. Figure 4-3 shows an enlarged view of each of the feature datasets and their associated feature classes. 

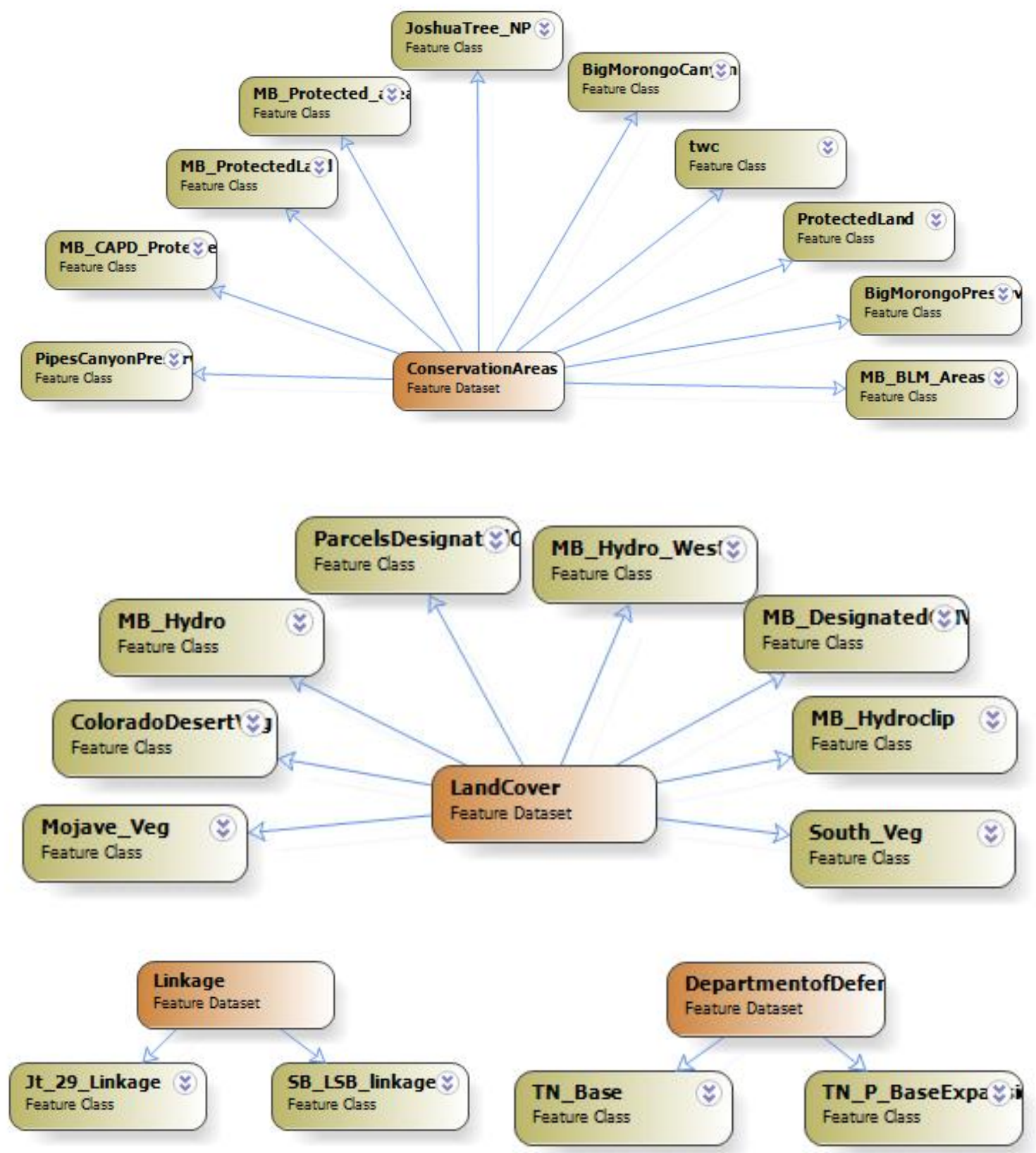



\section{Figure 4-3: Feature Datasets and Feature Classes}

Many of the datasets were reduced to binary or transformed to derived values within the parcel dataset. Any original information about those data would have to come from the original dataset and therefore these were retained. For example, the road density was calculated from transportation dataset and was added to the parcel feature class, while the original transportation data were maintained in the geodatabase available for use in future projects and analysis.

The final decision that was made was the determination of what data model would be used in the project. Either vector or raster data models could have been used to perform the weighted overlay analysis of this project, but the raster data approach would have lead to several problems in this project because of inherent issues in raster data. Raster data is composed only of pixels that can contain only one attribute per feature. The 
conversion of vector to raster data has the benefit of increased processing time but shape, identity, and the attributes of the point line or polygon are diminished or lost.

For example, the conversion of vector data to raster data is dependent on the cell size. If vector data is processed as raster data the chosen pixel size has to maintain the shape of the feature. Using too large pixel size will cause information to be lost and too small cell size will use more storage space and increases processing time without necessarily adding accuracy. In addition, data can be misrepresented because each pixel can only represent one value. For example, the boundary of a parcel may run across the middle of a cell. That cell will be given the value of the largest fraction of the cell of the middle point of cell. Furthermore, data the result will most likely have irregular shapes or slivers due to the conversion integer data, such as the suitability score of each cell, back to vector. These features, created by the raster-to-vector conversion, would be hard to relate as attributes of a vector data set such as the parcel data set. These slivers would cause issues in attempting to calculate the suitability score of each parcel and introduce an amount of error that would not be acceptable for this project.

Another drawback of converting all of the data into raster data is that it would reduce the practical modifiability of the model. Because raster data can only maintain one attribute per feature, each dataset has to be simplified down to the one criterion that needs to be evaluated. That data is then reclassified so that is can be evaluated in the weighted overlay. That conversion leads to the loss of a significant amount of information about the data set. In addition, that converted raster data set is static. Without the use of programming and complex geoprocessing, the only element the end user could manipulate is the weight of the reclassified data set. The end user would lose control of the classification process. This classification process is important in this project because there are no standards to base the development assumptions on.

Standards are available to many suitability analysts and define the classification. These standards could be replaced by research or expert opinion. In this case the client could have been considered an expert but the client did not feel confident with making these decisions arbitrarily. To mediate this issue, the client could proceed through the model with a trial and error approach to setting classes, but this trial and error process cannot be easily done with raster data unless every iteration of the process is done from scratch in the vector to raster conversion process or done through complex geoprocessing. It was decided that the analysis be done in the vector data model because of these issues.

The drawback of performing these spatial analyses in the vector data format was that the model development process did not have the added benefit of using Spatial Analyst tools available with ArcGIS. As a result, the end product had a more complicated interface and was more difficult to manipulate, maintain, and run. This was outweighed by the added flexibility, modifiability, and repeatability.

In order to optimize the model development process, all of the criteria datasets were simplified to be attributes of each data set. This data processing is described in detail in 
Chapter 5. Once the decision was made to maintain the vector data model throughout this project, the database was designed to organize and store the data essential in this project.

\subsection{Data Sources}

The essential data sets in this project were defined through client conversations and research. These datasets were then obtained and organized in the geodatabase. Prior to the development of the geodatabase all of the needed datasets were enumerated and described in the Master Input Data List (MIDL) (Table 2).

Table 2. Morongo Basin Conservation Suitability Model MIDL

\begin{tabular}{|c|c|c|c|}
\hline Data Set Name & Description & Data Source & $\begin{array}{l}\text { Data Processing } \\
\text { (if available) }\end{array}$ \\
\hline $\begin{array}{l}\text { California Protected Areas } \\
\text { Data }\end{array}$ & $\begin{array}{l}\text { Polygon data set of the } \\
\text { conserved lands within the } \\
\text { state of California. These } \\
\text { conserved lands included } \\
\text { BLM, NPS, DOD, State } \\
\text { Lands Commission and } \\
\text { privately conserved lands }\end{array}$ & $\begin{array}{l}\text { Provided by the client } \\
\text { originally compiled by } \\
\text { CPAD }\end{array}$ & $\begin{array}{l}\text { Had to be clipped to the } \\
\text { focus area and a layer had } \\
\text { to be created containing } \\
\text { none of the Undesignated } \\
\text { BLM areas. }\end{array}$ \\
\hline MB Parcels & $\begin{array}{l}\text { Polygon dataset of the } \\
\text { Morongo Basin parcels. }\end{array}$ & $\begin{array}{l}\text { Provided by the client } \\
\text { originally available from } \\
\text { the county of San } \\
\text { Bernardino }\end{array}$ & $\begin{array}{l}\text { Had to be processed to } \\
\text { contain information of } \\
\text { each of the evaluation } \\
\text { criteria as an attribute of } \\
\text { the dataset. }\end{array}$ \\
\hline Base Expansion Plans & $\begin{array}{l}\text { Polygon dataset of the } \\
\text { areas proposed for the } \\
\text { Twentynine Palms Marine } \\
\text { Base Expansion. }\end{array}$ & $\begin{array}{l}\text { Provided by the client with } \\
\text { the authorization of the } \\
\text { MCAGCC }\end{array}$ & \\
\hline MB Vegetation & $\begin{array}{l}\text { Polygon data set that is a } \\
\text { compilation of the best } \\
\text { available vegetation data } \\
\text { sets. }\end{array}$ & $\begin{array}{l}\text { Obtained from the } \\
\text { California GIS database } \\
\text { developed by the } \\
\text { California Resources } \\
\text { Agency }\end{array}$ & $\begin{array}{l}\text { The data set had to be } \\
\text { clipped to the focus area }\end{array}$ \\
\hline $\begin{array}{l}\text { BLM Designated OHV } \\
\text { Routes }\end{array}$ & $\begin{array}{l}\text { Line feature class that } \\
\text { shows the areas that have } \\
\text { been designated as legal } \\
\text { OHV routes. }\end{array}$ & $\begin{array}{l}\text { Provided by the client } \\
\text { originally developed by the } \\
\text { BLM }\end{array}$ & $\begin{array}{l}\text { Had to be clipped to the } \\
\text { focus area }\end{array}$ \\
\hline Jt 29 Linkage & $\begin{array}{l}\text { Polygon feature class } \\
\text { showing the least cost } \\
\text { paths for wildlife } \\
\text { movement. }\end{array}$ & $\begin{array}{l}\text { Provided by the client with } \\
\text { the authorization of the } \\
\text { SCW }\end{array}$ & \\
\hline Tiger Roads & $\begin{array}{l}\text { Line feature class of the } \\
\text { roads in the Morongo } \\
\text { Basin }\end{array}$ & $\begin{array}{l}\text { Provided by the client but } \\
\text { had to be replaced } \\
\text { because of missing } \\
\text { metadata }\end{array}$ & $\begin{array}{l}\text { The dataset obtained had } \\
\text { to be clipped to the focus } \\
\text { area. }\end{array}$ \\
\hline MB_Hydro & $\begin{array}{l}\text { Line feature class of the } \\
\text { hydrographic features in } \\
\text { the Morongo Basin. }\end{array}$ & $\begin{array}{l}\text { Provided by the client } \\
\text { USGS NHD dataset that } \\
\text { was adapted for the client } \\
\text { by Green Info Network. }\end{array}$ & \\
\hline
\end{tabular}


The client provided all of the data she had regarding the area. These datasets were compiled from various sources and had varying degrees of documentation. Of the datasets only the roads data provided by the client was unusable. It was necessary to find the lineage for this dataset and replace it before loading it in the database. The reason this dataset was replaced was because it was important to know the source, accuracy, codes, and intended use of the datasets before the project was started.

\subsection{Data Collection Methods}

The client provided all of the datasets necessary for the completion of the project except for a quality roads dataset. The only data collection was done to replace the roads dataset that had no metadata or documentation. The roads dataset was determined to be TIGER roads and was obtained from the United States Census Bureau web site.

\subsection{Data Scrubbing and Loading}

The required datasets for this project covered a range of geographic areas as well as projections. In order to load, into the database, these issues had to be corrected.

The projection for this geodatabase was determined to be the NAD 1983 UTM Zone $11 \mathrm{~N}$. This system was chosen because the Joshua Tree National Park, as well as the South Coast Missing Linkage project, was based on it. The data that were not in this projection were reprojected and then loaded into the database. In addition, the focus area for the MBOSG is defined by the Morongo Basin Unified School District boundaries. For the purposes of this project, all of the datasets were clipped to this boundary. Once the data preparation process was complete, these datasets were loaded into the designed ArcGIS file geodatabase. In addition to the clipped focus area data sets, the original data sets were also loaded into a geodatabase for the use in future projects (Figure 4-4).

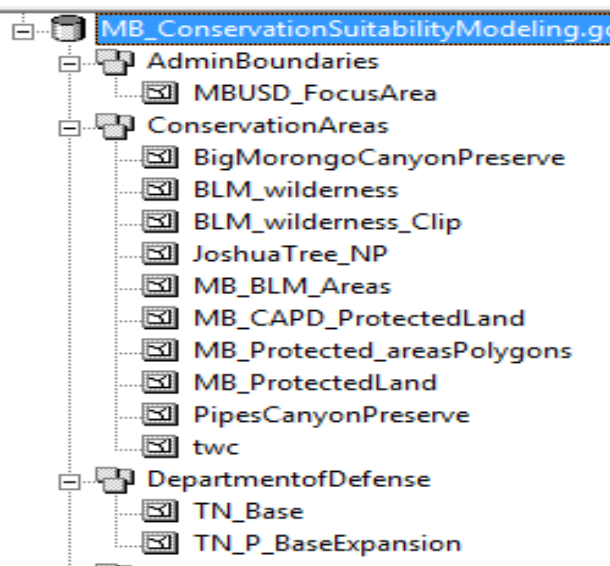

Figure 4-4: Final Geodatabase 


\subsection{Summary}

The conceptual data model explained that all of the data that was involved in this project were essentially features of the parcels. Each parcel interacted or contained the criteria for conservability of that particular piece of land. A vector data model was determined to be the format on which the project would be based. This decided the type of schema to be used in the geodatabase. After these decisions were made, the essential data sets identified by the client were compiled and loaded into the database. The client provided many of the datasets and very little processing had to be done to load the data into the geodatabase. The primary processes that were completed to get the data into the geodatabase were projecting the data into the geodatabase projection and clipping it to the project focus area. 


\section{Chapter 5 - Implementation}

The Morongo Basin Conservation Suitability Model project made the spatial information more interpretable to the client, which aide in action plan development. The project utilized the ArcGIS software with ModelBuilder and scored the basin's parcels based on their suitability for conservation. The first process that had to be completed was the conservation suitability criteria evaluation. This process was followed by the preparation of the parcel dataset for use in the model by including the criteria attributes to the dataset. After the parcel dataset was prepared, the model was developed with the available tools from ArcGIS and custom tools developed using Python scripting.

\subsection{Criteria Identification and Analysis}

The suitability of a parcel for conservation is influenced by many physical and environmental factors. Many of the environmental factors were taken into consideration by the South Coast Wildland's (SCW) Linkage Design to evaluate suitable areas for species movement. Because these factors were included in their analysis and the Linkage Design was taken into account in this analysis, they were not included to avoid the double counting. In addition to the factor of the interaction with the linkage design, other factors that contributed to conservation suitability were also considered, including parcel size, vacancy, nearness to currently conserved areas, presence of Off Highway Vehicle (OHV) routes, and density of roads and hydrographic features. After consulting with the client these criteria were then classified into five categories: less suitable, moderately suitable, suitable, highly suitable, and extremely suitable (Table 3). For example, a parcel that is smaller than 15250 square meters was considered less suitable for conservation purposes, while a parcel that is larger than 1,700,000 square meters was regarded as extremely suitable. Since these class break values for the classes are subjective, they can be redefined in the model. 
Table 3. Classification Criteria

\begin{tabular}{|c|c|c|c|c|c|}
\hline Layer name & Less Suitable & Moderate Suitable & Suitable & High Suitable & Extremely Suitable \\
\hline & 1 & 2 & 3 & 4 & 5 \\
\hline Vacant & No & & Yes & & \\
\hline Linkage Design Interaction & No & & & & Yes \\
\hline Hydrography Density & $<0.000319$ & 0.000864 & 0.001472 & 0.002436 & $>0.002437$ \\
\hline Road Density & $>0.008256$ & 0.008255 & 0.005931 & 0.003141 & $<0.001266$ \\
\hline Parcel Size & $<15250$ & 125000 & 550000 & 1700000 & $>1700001$ \\
\hline Nearness to Conservation Areas & $<1609$ & 3218 & 8046 & 11265 & $>11266$ \\
\hline OHV Designated Routes & Yes & & No & & \\
\hline & & & & & \\
\hline
\end{tabular}

In addition, the client instructed that some parcels should be restricted from the evaluation, including currently conserved areas, areas planned for development, and areas considered for base expansion. These areas would not be assessed in the final analysis because they are not available for conservation opportunities.

Before the classification can be performed, the data were processed and incorporated as part of the parcel dataset. Put differently, all of the factors had to be attributes within the parcel dataset. The next section explains the detailed procedures for data preparations.

\subsection{Data Preparations for Model Use}

The data model that was used for this project was the vector data model as opposed to the raster data model. Since ESRI ArcMap has no weighted overlay tools for vector data, special procedures had to be taken to prepare the data for use in the vector data weighted overlay process. It was decided that to perform the weighted overlay all of the aforementioned factors had to be attributes within the parcel dataset. However, many of the identified factors, such as road density, were not part of the original parcel data. In order to get these criteria factors into the parcel dataset, the factors had to be evaluated and assigned an Assessor Parcel Number (APN) to link to the parcel.

The datasets that were processed and included as attributes of the parcel dataset were: conservation areas, base expansion plans, road and hydrology datasets, BLM designated OHV routes, the linkage design, and areas planned for development. To derive the required attributes from these datasets and append these attributes to the parcel data, various spatial operations were used, which will be described as follows. 
Although the existing conservation lands dataset was at its most basic unit composed of parcels, none of the areas had parcel numbers associated with them. The parcel dataset and the conservation area boundaries are exactly coincident so the issue of a single parcel having multiple conservation areas within it was not an issue. For this reason, an intersect tool was used to join the parcel dataset to the conservation lands dataset. The intersect tool computes all of the layers that overlap and writes the associated attributes to the final output. Therefore, after this spatial operation conducted, all the parcels that overlap with conservation lands had all the attributes from the conservation lands data. A calculation was further performed on this resulting output by coding the parcels that fall in conservation lands as "yes" and others as "no". This attribute field was then added to the original parcel data, as well as the management status of the land. The management status of the land was used for analysis purposes after the suitability score was calculated. The same process was followed to incorporate the base expansion plans in the parcel dataset.

In contrast, the spatial join tool was used to determine the interaction of parcels with the suitable areas for species movement from SCW's Linkage Design and BLM designated OHV routes. This tool was used because it links all parcels with these two datasets as long as the parcels have some overlap or shared boundary with these features. In the resulting output, a field called "joint_count" was generated, which indicates the presence of a joined feature. Based on this value, a new field was calculated to indicate "yes these parcels interact", or "no they do not interact". The output was then joined to the project parcel dataset and new attributes indicating whether parcels interact with the Linkage Design and OHV routes were added.

The roads and hydrographic features were processed with custom tools because of some deficiencies in the ArcGIS software. This process was the most time consuming and difficult. It is possible to calculate the density of lines using the spatial analyst tools in ArcGIS. This output, however, would be a raster surface and could not be attributed to the parcels accurately. It was decided that the density be calculated for each parcel individually. Because parcels do not typically have a significant number of roads that bisect them, the density was calculated for a buffer area around each parcel. It was determined that the density would be calculated for a 500 meter buffer around each parcel. This distance was determined somewhat arbitrarily because of the deficiencies of the ArcGIS software. The line features were then intersected with the buffer area. This was done to dissect the roads into line segments within the buffered area. The line segments were then summed up for each buffered area and the linear meters of the line segments divided by the area meters of the buffer.

The ArcGIS program could only handle processing 500-1000 parcels at a time; any more than that the processes would stall and return this error message (Figure 5.1). As a result, this process was automated using Python scripting because the data had to iterate through the parcels. The Python scripting code is in Appendix A. 


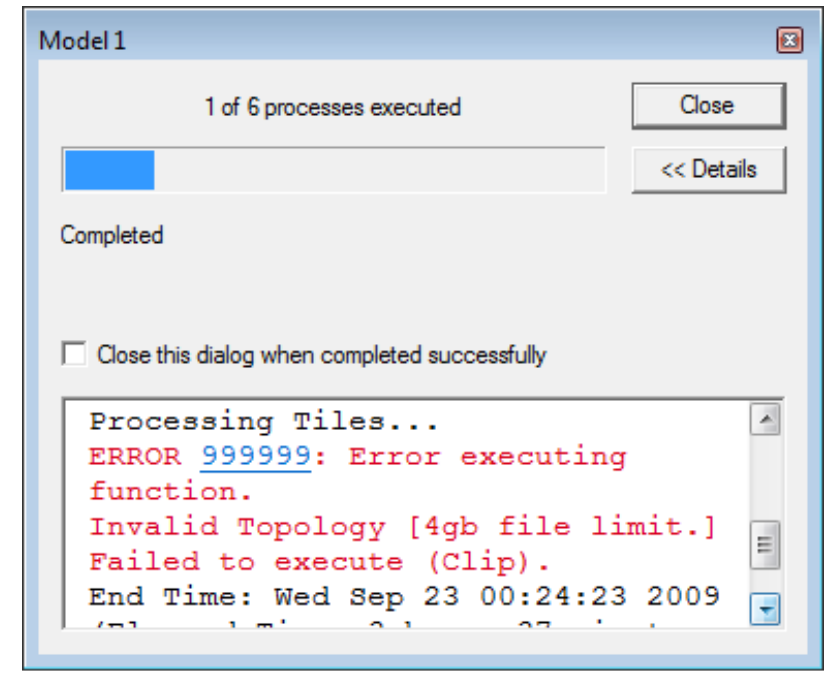

Figure 5-1: Density Calculation Error Message

If a small buffer size was used, the program could process through without error but the results would have been useless. The smallest functional buffer size was 500 meters and worked with a batch size of 500 or 1000 . A larger buffer size could be processed if needed using the custom tool but the batch size would have to be reduced to even smaller size to process without error. For this initial analysis, the 500 meter buffer size was sufficient. The client can adjust the buffer size for the density tool. The client would just have to buffer the parcels with the desired buffer distance and input that buffer distance in the tool.

Each buffer retains the APN of the parcel that it is associated with and can be joined back to the project parcel dataset. A field was created in the parcel dataset for the road and hydrographic density and calculated from the joined 500 meter buffer dataset. If a different buffer distance is desired, the client can calculate it using the tool, join that dataset, and replace the original density values or add a new field for the new buffer distance density.

The near tool was used to determine the nearness of the parcels to currently conserved areas. The near tool calculates the distance in map units of the input feature (Parcels) to the near features (conserved areas) and adds the fields Near_ID and Near_Dist to the input dataset. The Near_Dist attribute is the distance that was used in the suitability score analysis. All of the conservation areas were added to the analysis. These excluded the BLM management areas that were not designated wilderness. The BLM lands in the Mojave Desert are currently under threat to renewable energy development due to their multiple use designation. For this reason these lands are not considered truly conserved by the regional conservation agencies.

The vacancy of the parcel and whether that parcel was planned for development were also processed. The parcel data set has an attribute field called LandUseCode; one of the 
land use codes is vacant. With the use of aerial imagery, available from ESRI base data, this code was evaluated for accuracy. Many of the parcels that had a land use code of null were actually vacant. Using heads up digitizing methods, the null parcels that were vacant were corrected in the dataset. After that process was completed a, binary field was created and coded as "yes" if the parcel was vacant and "no" otherwise. Whether the parcel was planned for development required the incorporation of the county or city development excel files into the spatial parcel dataset. This process was done by the client and then joined to the project parcel dataset. A binary field was then added, the value being "yes" for the parcel that was planned for development or "no" for the parcel that was not planned for development.

The final evaluation factor, the parcel size, did not have to be added to the parcel dataset, as it is already an attribute and can be used directly in the evaluation model. After all of these processes were done the project dataset was purged of all the unnecessary attribute fields and was ready for use in the model development.

\subsection{Model Development and Spatial Analysis}

In this project the spatial analysis was performed using ArcGIS ModelBuilder that is available with ArcInfo license. The models were represented as classification, weighting, and final scoring. Each of the relevant factors were classified and the classification was based on their relative suitability for conservation. These factors were then weighted according to their importance in assessing the suitability of the land. The weight was indicated as a multiplier in the process as a fraction of 100. This process was illustrated using Excel. The Excel spread sheet was used in order to calculate the values of the weighted criteria and the multiplier used in the evaluation (Table 4). 


\section{Table 4. Weight Calculation Spreadsheet in Excel}

\begin{tabular}{|c|c|c|}
\hline Layer name & Assigned Weight & Percent Weight \\
\hline Planned for Development & Restricted & \\
\hline Currently Conserved & Restricted & \\
\hline Base Expansion Plans & Restricted & \\
\hline Vacant & 3 & 21.42857143 \\
\hline Linkage Design Interaction & 3 & 21.42857143 \\
\hline & & $\mathbf{0}$ \\
\hline Hydrography Density & 2 & 14.28571429 \\
\hline Road Density & 2 & 14.28571429 \\
\hline Parcel Size & 1 & 7.142857143 \\
\hline Nearness to Conservation Areas & 2 & 14.28571429 \\
\hline OHV Designated Routes & 1 & 7.142857143 \\
\hline & \multicolumn{2}{|c|}{14100} \\
\hline
\end{tabular}

This spreadsheet calculated the weight values and the values were need to be imported to the geodatabase in a table format. In doing so, weight values can be linked to the model using Python. This has been done so the end user could adjust the weights and classifications in the table and the values will be automatically adjusted within the ModelBuilder process (Figure 5-2). 


\begin{tabular}{|c|c|c|c|c|c|c|c|c|c|c|}
\hline & OBJECTID* & Title & FieldName & Unsuitable & LessSuitable & Moderate & Suitable & HighSuitable & Scale0rder & Weight \\
\hline & 1 & Planned for Development & PlannedDev & 1 & 0 & & & & BOOL & \\
\hline & 2 & Currently Conserved & Conservati & 1 & 0 & & & & BOOL & \\
\hline & 3 & Base Expansion Plans & BaseExpans & 1 & 0 & & & & BOOL & \\
\hline & 4 & Vacant & Vacantlots & & 0 & & 1 & & BOOL & 21 \\
\hline ) & 5 & Linkage Design Interaction & Linkage_De & & 0 & & & & BOOL & 21 \\
\hline & 6 & Hydrography Density & Hydro_dens & & 0.000319 & 0.000864 & 0.001472 & 0.002436 & ASC & 14 \\
\hline & 7 & Road Density & RoadDensit & & 0.008256 & 0.005931 & 0.003141 & 0.001266 & DESC & 14 \\
\hline & 8 & Parcel Size & ParcelSize & & 15250 & 125000 & 550000 & 1700000 & ASC & 8 \\
\hline & 9 & Nearness to Conservation Areas & NEAR_DIST & & 11265 & 8046 & 3218 & 1609 & DESC & 14 \\
\hline & 10 & OHV Designated Routes & OHV_Intera & & 1 & & 0 & & BOOL & 8 \\
\hline
\end{tabular}

\section{Figure 5-2: Criteria Classification Geodatabase Table}

The classes and weights represented above were used in the original prioritization of the parcels (Table 3 and Figure 5-2). The classification of each factor was mapped to visualize each of the factors that were going into the model, as well as visualize the class break points of the criteria sets that were comprised of ranges. These criteria maps are demonstrated in a series in Figure 5-3. 

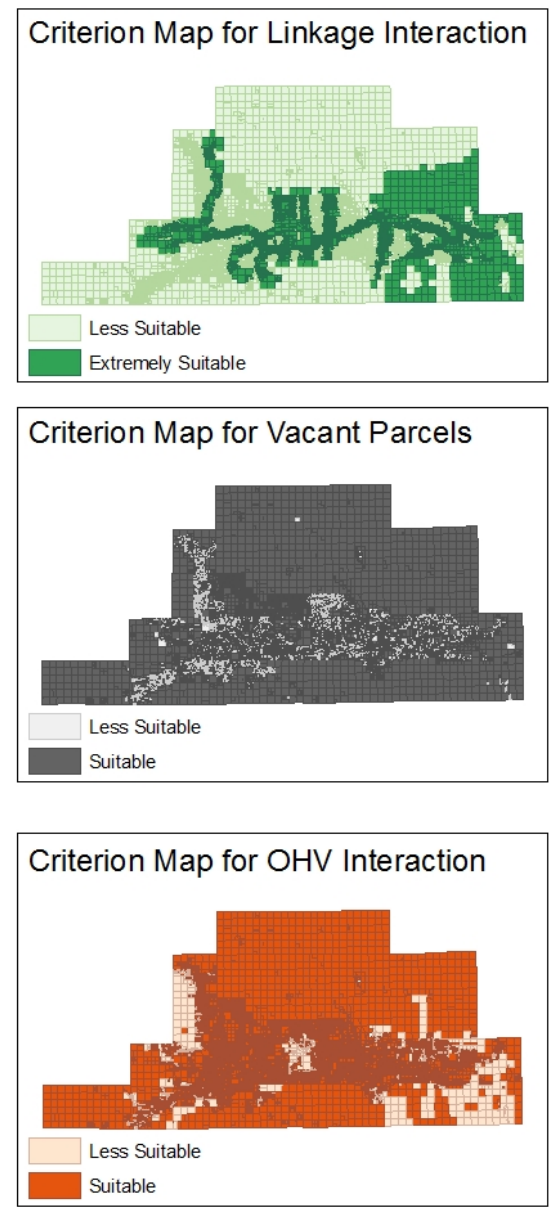
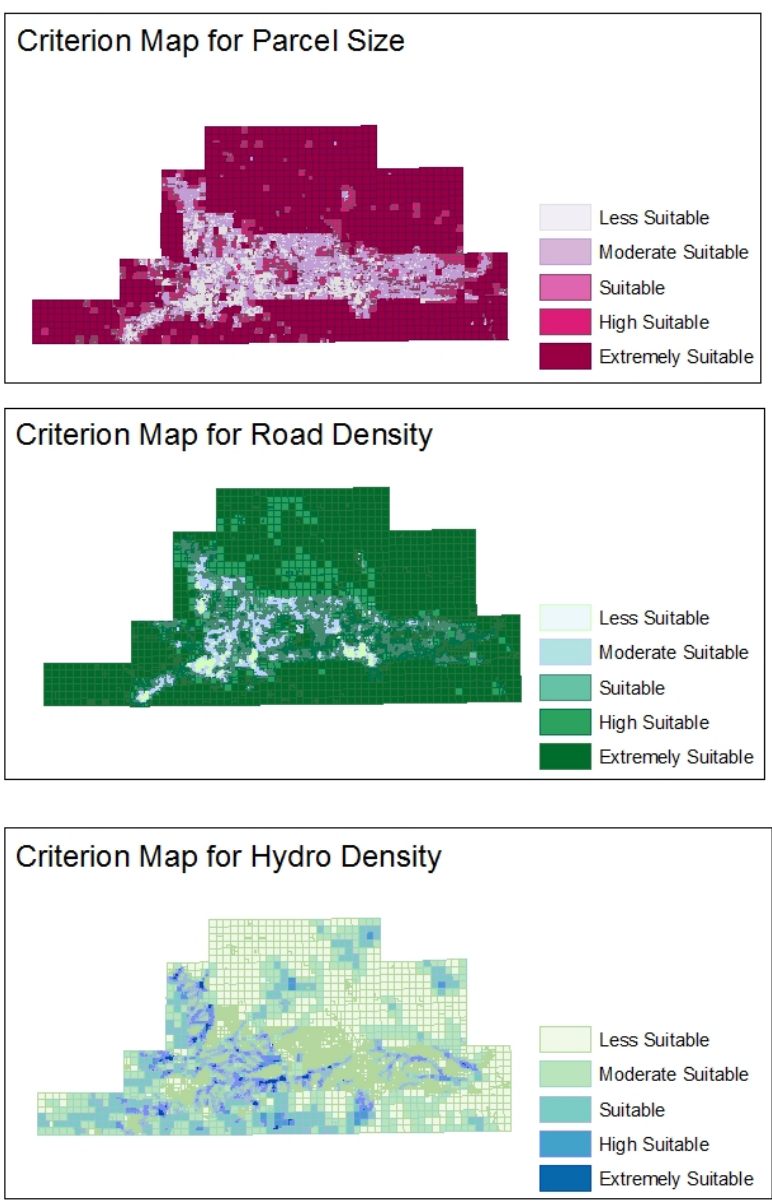

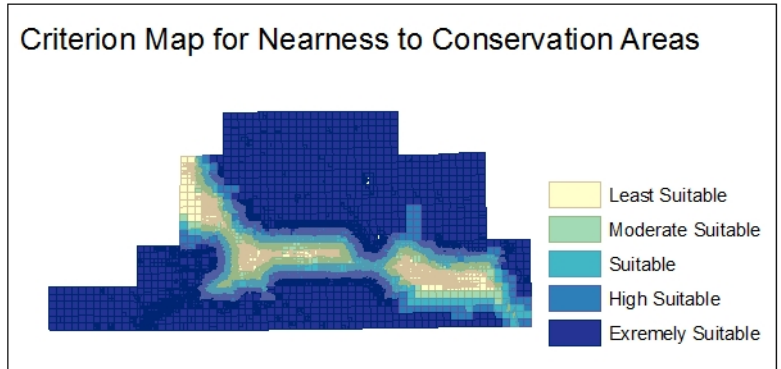

\section{Figure 5-3: Classified Layer Maps}

The model was broken down into three parts: the addition of the reclassification field named "reclass"; the addition the weight field and score calculation; and the addition of the final suitability score and calculation.

The first part of the model was the addition of the classification field and the calculation of that field (Figure 5-4). This process adds the "reclass" field and then calculates the field based on the classes defined in the classification table (Table 3). The 
table as explained above was linked to the model using Python scripting so that the process could be automated. An alternative was for the user to select the classes of each factor and calculate the field for each class 1 thru 5 in Modelbuilder. This approach would be cumbersome and time consuming; especially the user needs to know how to edit parameters in Modelbuilder. This decision to use Python scripting to link the Excel spread sheet was made so that the end user only need to change the parameter in Excel and the model will update with new classification criteria.

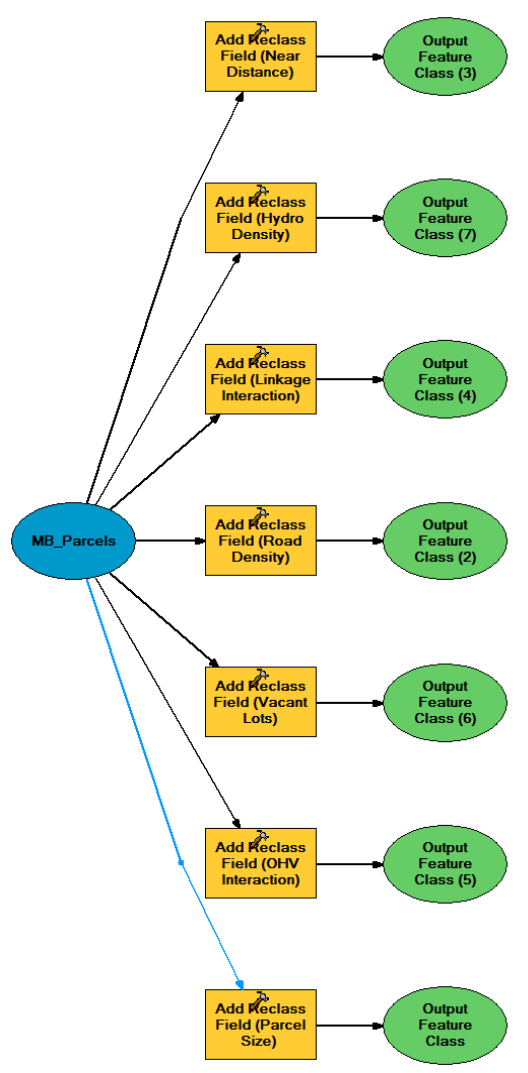

Figure 5-4: ModelBuilder Addition of a Reclass Field

The second step of the suitability model was the addition of the weight and score field for each of the factor (Figure 5-5). For a clear view of the model, please refer to Appendix B. 


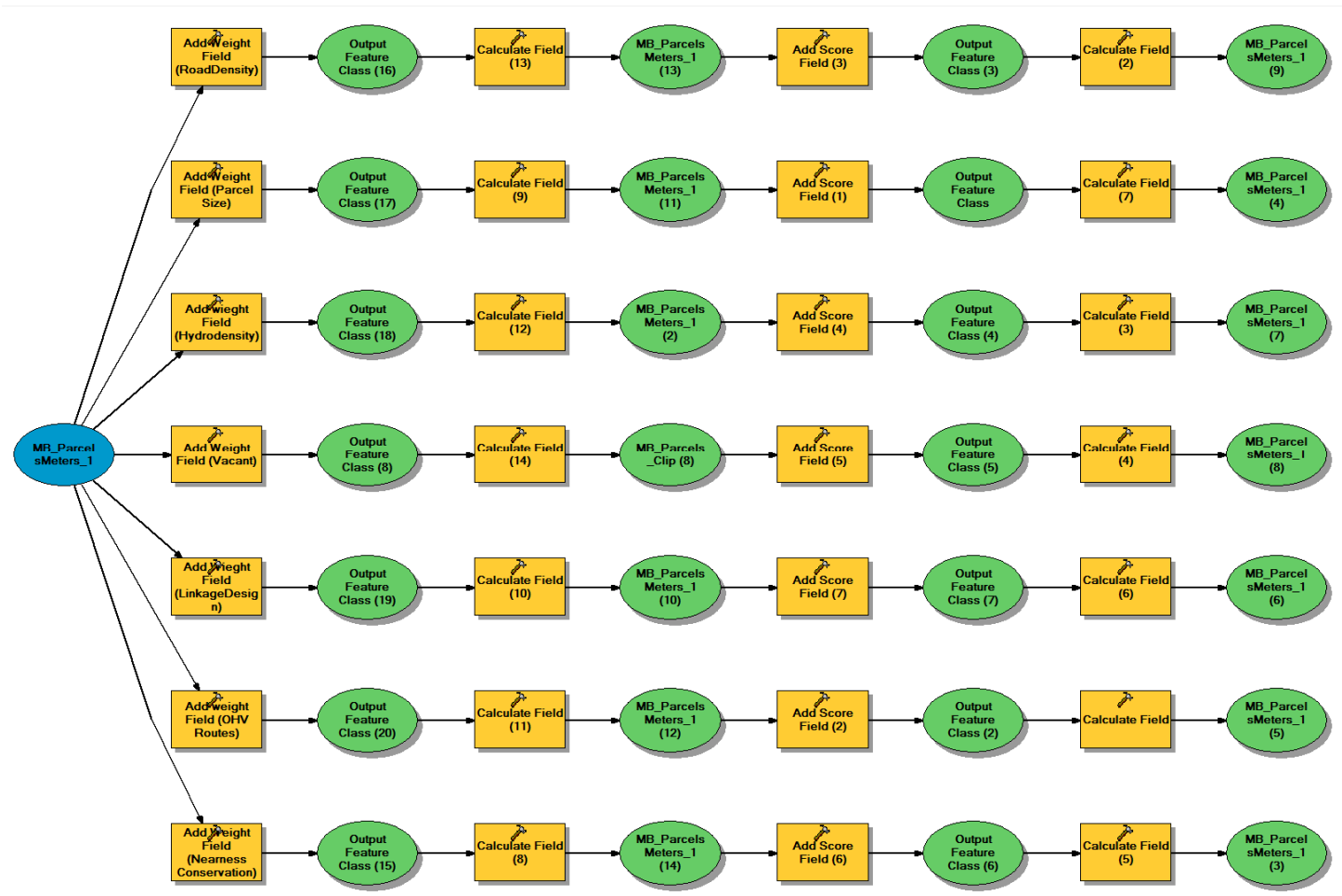

Figure 5-5: ModelBuilder Criteria Weighting and Scoring

The weight of each of the factors is dependent on the weight determined in the weighing Excel spread sheet (Table 4). The weights were based on a 1-3 score. This scoring was decided on because of its simplicity: 1 least important, 2 important and 3 most important. The scores of all of the factors were added up to get the sum of the weights of 14. Each weight was then transformed to the weights based on the scale of 100 (the "percent weight" field in Table 4). The scale of 100 was decided on because of the familiarity of a 100 percent scale. Each of the percent weights adds up to 100 percent of the suitability. Finally, the multiplier was calculated by dividing the percent weight by 100 for each factor, which was used to compute the suitability scores for each factor.

The final score of each of the factors was calculated by multiplying the class value of the factor with the corresponding multiplier. For example, if a parcel is vacant, it is suitable for conservation and therefore the class value is 3 . With its multiplier of 0.21429 , it contributes 0.6428 to the final suitability score of the parcel.

The final step of the ModelBuilder process was the calculation of the final suitability score (Figure 5-6). 


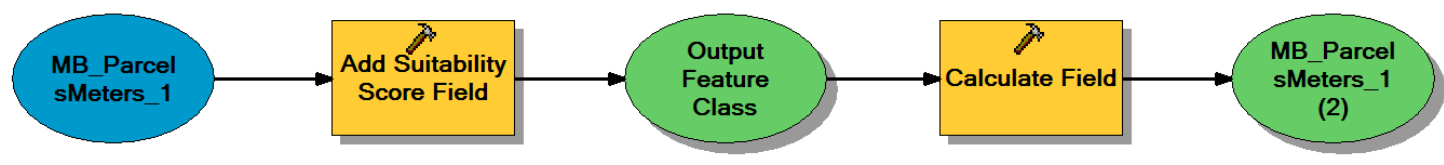

\section{Figure 5-6: ModelBuilder Final Suitability Score Calculation}

The calculation of the suitability score was simply the process of adding all of the individual criteria scores calculated in the previous step of the model. For example, if a parcel obtained an "extremely suitable" score of five for all of the factors, it would have a final score of five. In the initial run of the model, this score was impossible to obtain because several of the binary criteria only had a highest score of three. In later analyses, this could be changed if the client classified the factors differently. With the application, the client could adjust the classification and weights.

As mentioned earlier, some factors determine that a parcel is not possible for conservation plan, such as the areas that have already conserved. The parcels that meet these restricted factors were then, using a SQL query, were classified as zero because they have no potential for being conserved or considered for stewardship efforts. As such, they were excluded for the initial run of the model. However, the client considered including these datasets in the evaluation so that the option of including them is possible in future runs of the model.

The final output was then mapped so that the client and members of MBOSG could visualize the potential of this analysis. In addition, the end user could produce a report using the results and possibly query those results based on any of the conditions they desired.

The end user will not interact with the above model diagrams. The end user need only to interact with the criteria parameters table (Figure 5-4) and the calculate suitability score tool (Figure 5-7). The end user will input the prepared project parcel dataset and the criteria breaks table and specify the output feature class name and location then run the tool. Python scripting was used to automate the ModelBuilder process that was described above. 


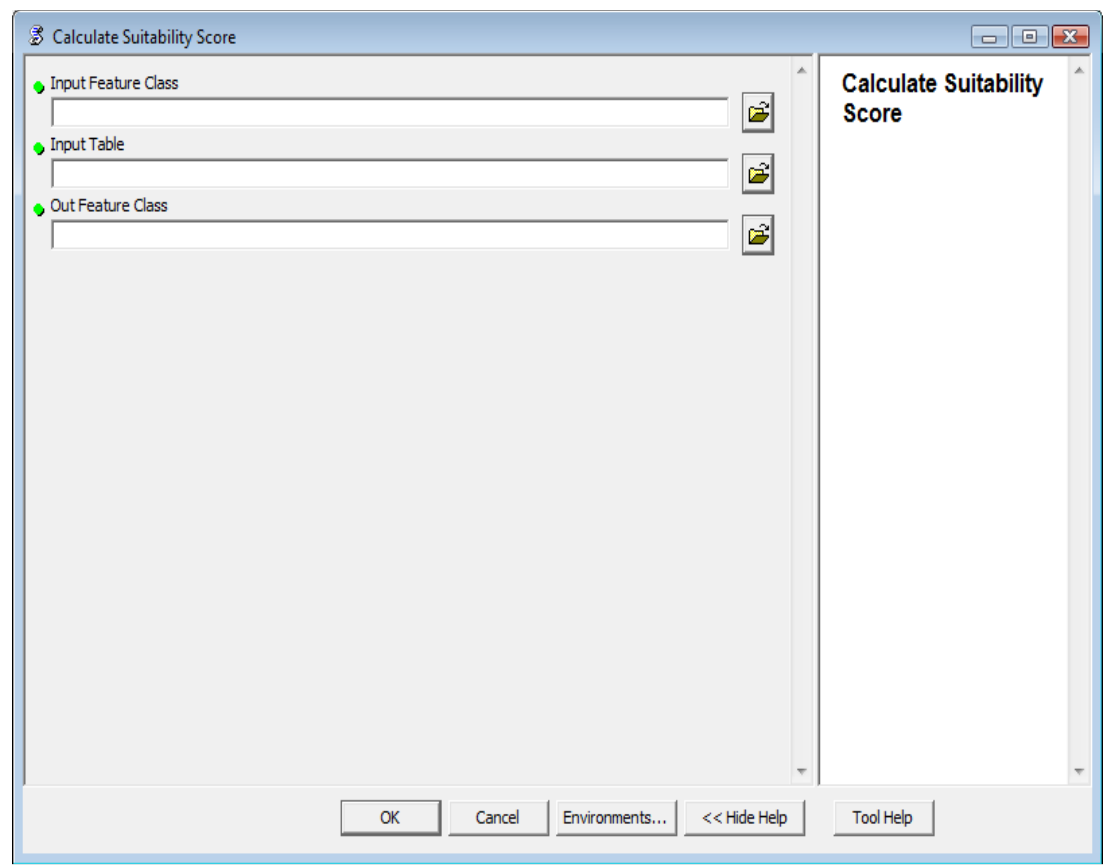

Figure 5-7: Calculate Suitability Score tool user interface

\subsection{Summary}

Prior to the ModelBuilding process, of the evaluation factors had to be processed to be attributes within the project parcel dataset. Deriving these attributes had varying levels of processing and some required the development of a model to process the dataset. After these factors were processed to be attributes, the model could be developed. The model building was comprised of three parts: the calculation of the reclassification field, the weighting and scoring of each of the criteria, and the summing to the scores into a single suitability score. After the suitability score was calculated, the parcel dataset could be analyzed for the purposes of conservation action planning. 


\section{Chapter 6 - Results and Analysis}

This chapter will describe several topics: the original parameter and the results; the potential ways and reasons for modification of the parameters; and examples of several potential analyses done with the final results.

\subsection{Representing the Model Results}

The model was run several times to test the results and visualize how different factors affected the final results. The original classification schemas described in Chapter 5 (Table 3 and 4 ) were used in the initial run of the model. To remind the reader of the parameters, Figure 6-1 displays them again here.

\begin{tabular}{|c|c|c|}
\hline Layer name & Less Suitable & Moderate Suitable \\
\hline & 1 & 2 \\
\hline Vacant & No & \\
\hline Linkage Design Interaction & No & \\
\hline Hydrography Density & $<0.000319$ & 0.0 \\
\hline Road Density & $>0.008256$ & 0.00 \\
\hline Parcel Size & $<15250$ & 12 \\
\hline Nearness to Conservation Areas & $<1609$ & \\
\hline OHV Designated Routes & Yes & \\
\hline Layer name & Assigned Weight & Percent Weight \\
\hline Planned for Development & Restricted & \\
\hline Currently Conserved & Restricted & \\
\hline Base Expansion Plans & Restricted & \\
\hline Vacant & 3 & 21.4 \\
\hline Linkage Design Interaction & 3 & 21.4 \\
\hline & & 0.0 \\
\hline Hydrography Density & 2 & 14.3 \\
\hline Road Density & 2 & 14.3 \\
\hline Parcel Size & 1 & 7.1 \\
\hline Nearness to Conservation Areas & 2 & 14.3 \\
\hline OHV Designated Routes & 1 & 7.1 \\
\hline & 14 & 4100 \\
\hline
\end{tabular}

\section{Figure 6-1: Initial Parameters}


The initial parameters were determined through discussions with the client. Some of these parameters were set arbitrarily significant consideration was given to their classification and weighting. After the classification and weighting were completed, the parameters were entered into the model and the model was run. The final result of the model is a suitability score that is an attribute within the parcel dataset and can be symbolized to show areas of more or less suitable for conservation (Figure 6-2).

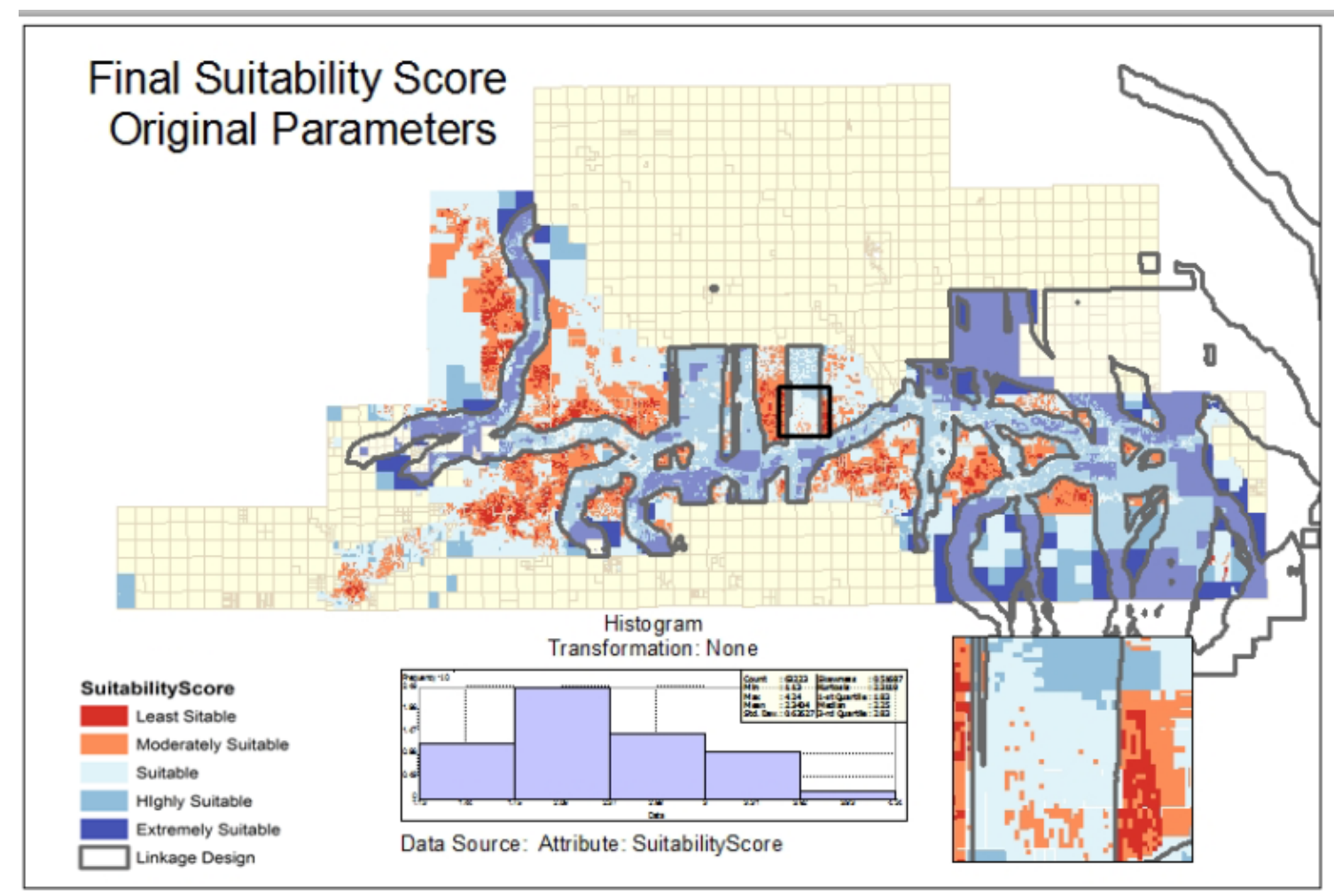

Figure 6-2: Final Suitability Score Using Initial Parameters

This choropleth map can be displayed differently with the various classification methods. The benefit of changing the classification breaks is that different classification methods can show patterns in the data that could not be seen with another method. It is the decision of the end user or analyst of the data to determine the classification method for the data. It is recommended that the user use either equal interval classification or manual breaks method.

It was decided that the equal interval classification method should be used in the analysis of the results because it has intuitive class breaks that are determined by the value of the attribute. This emphasis on the attribute value is important because of the nature of the data. The intuitive class breaks are helpful when explaining the data to unfamiliar viewers. The natural breaks classification was adequate in classifying the data because it followed the pattern of the suitability however, the breaks were not intuitive. The manual breaks method can be applied to classify the data. However, the user should be familiar with the data and have clear goals in the analysis method. For this data, the 
manual classification can be time consuming. The quantile classification method should be avoided for use with the final results because the classes are misleading. The decision between the use of manual breaks and equal interval will ultimately be up to the end user. However, for the analyses in this report the final suitability score will be represented using equal interval classification.

\subsection{Model Results and Adjustments}

The final results of the model were determined to be acceptable. Areas that were developed obtained a lower score than the undeveloped areas. Areas within the linkage design scored higher than areas outside the linkage design. This is most likely due to the weight and the score of the linkage design criteria. The linkage design weight and score moderated the effects of the other factors considered. Despite the skew caused by this, there are several areas within the linkage design that do show low suitability scores. These areas are important for management purposes and a modification to the model weights should be used to make these areas more appropriately scored. One quality of the final result that could be interpreted as an issue is that the maximum suitability score possible is 4.429 , rather than 5 . The reason that the maximum score for suitability cannot possibly be five is because there are multiple binary criteria in which the maximum score is three, not five. These factors cause the maximum score possible to be 4.429 , as opposed to five, because the values are weighted, added, and averaged. If the end user sees this as an issue, these inputs can be modified in the model and those results can be determined to be acceptable or not. This issue should be mitigated by the equal interval classification method but the score can be easily recalculated. The model was designed to be modifiable so that it can adapt to best fit the needs of the analysis.

\subsubsection{Reclassifying Criteria}

The two factors taken into the model, including vacant and OHV interaction, was initially classified as binary, with the highest score being three. Because of these two datasets, the most suitable score for the final results could never be five. In order to see the effects these scores may have on the final result, these scores can be changed in the geodatabase table and the model rerun with the new values. Any changes to the geodatabase table will have to be done within a edit session in ArcMap.

Once the maximum class scores were changed from 3 to 5 for vacant parcels and $\mathrm{OHV}$ interaction, the model can be rerun and the final suitability scores were obtained. The change in the final results can be compared against the results of the original classification of the data (Figure 6-3). 

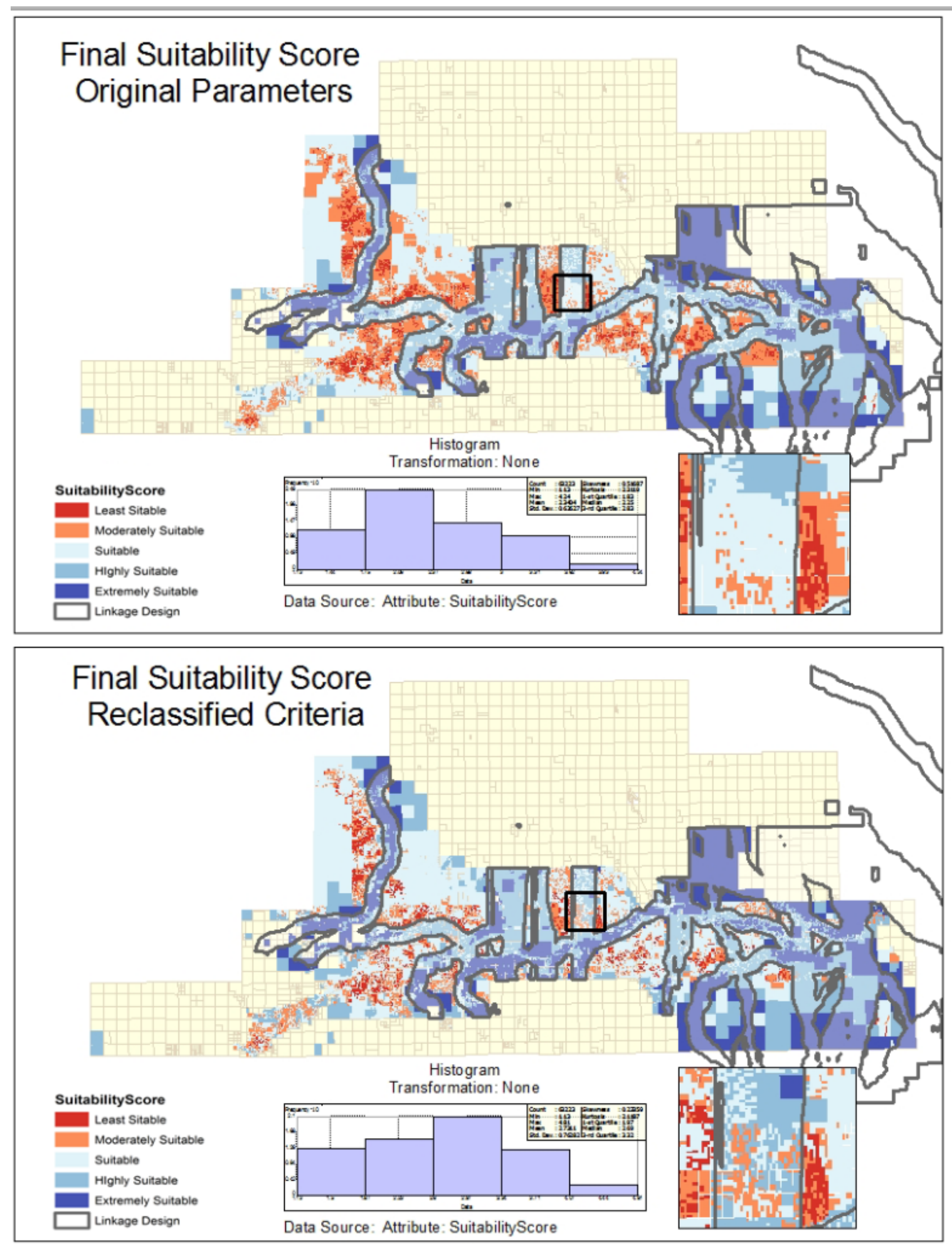

Figure 6-3: Reclassified Criteria Results 
The reclassification of these two factors had some effects on the distribution of the suitable parcels for conservation. These changes can be seen in the pattern of the choropleth map, as well as in the histogram of the data. The proportion of third class was increased, as were the two extreme classes. However, the second class was reduced. Because the vacant score was increased, all vacant parcels' suitability scores were increased as well. For example, vacant parcels that had high road density, small parcel size, and were not in the linkage design were classed as suitable even though they were negatively affected by those other features.

Every change in the model changes the final suitability sores. There are numerous ways that the classification of the data can be adjusted and it is impossible to explore every one of them. It will be the decision of the end user how to shift the classification of the data to meet their analysis goals.

\subsubsection{Adjusting Factor Weights}

Similarly, the model also allows the end user to adjust the weights assigned to each of the factors. These changes to the weights will be calculated within the Excel spread sheet and entered into geodatabase table then the model is rerun with the complete project dataset. There are various reasons the end user would want to change the weights from one analysis to another. The user may want to decrease the weight of the linkage design criteria to better estimate the effects of the other criteria on the suitability. In addition, the user may want to increase the weight of the criteria that may be seen as indicative of a greater opportunity for conservation; these criteria maybe nearness to conservation areas, parcel size, and vacancy. They may also want to evaluate the basin with all criteria considered equal.

In the original suitability results, the highly suitable areas generally followed the linkage design. In the original assessment, the parcels that interacted with the linkage design were classified as extremely suitable and weighted with the highest possible weight. Thus, it is difficult to prioritize parcels within the linkage design when a majority of those parcels are classified as highly or extremely suitable. The weight of this criterion can be adjusted so that it does not weigh on the results heavily (Table 5). 


\section{Table 5. Linkage Design Weights Adjustments Table}

\begin{tabular}{|c|c|c|}
\hline Layer name & Assigned Weight & - Percent Weight \\
\hline Planned for Development & Restricted & \\
\hline Currently Conserved & Restricted & \\
\hline Base Expansion Plans & Restricted & \\
\hline Vacant & & 14.3 \\
\hline \multirow[t]{2}{*}{ Linkage Design Interaction } & & 7.1 \\
\hline & & 0.0 \\
\hline Hydrography Density & & 21.4 \\
\hline Road Density & & 21.4 \\
\hline Parcel Size & & 14.3 \\
\hline Nearness to Conservation Areas & & 14.3 \\
\hline OHV Designated Routes & & 7.1 \\
\hline
\end{tabular}

In the example shown in Table 5, the weight of the linkage design was decreased and the weight of the vacant, road density, and hydro density were increased. The reason that these weights were increased was because in the absence of the linkage design factor, which considered all of these to some degree in its creation, these criteria become more important in the assessment. It is expected that this modification will not dramatically change the results with the exception of areas that have obvious conflicts with conservability, such as existing development. The results, when compared to the original parameters, show the expected change in the pattern of the suitability score (Figure 6-4). 

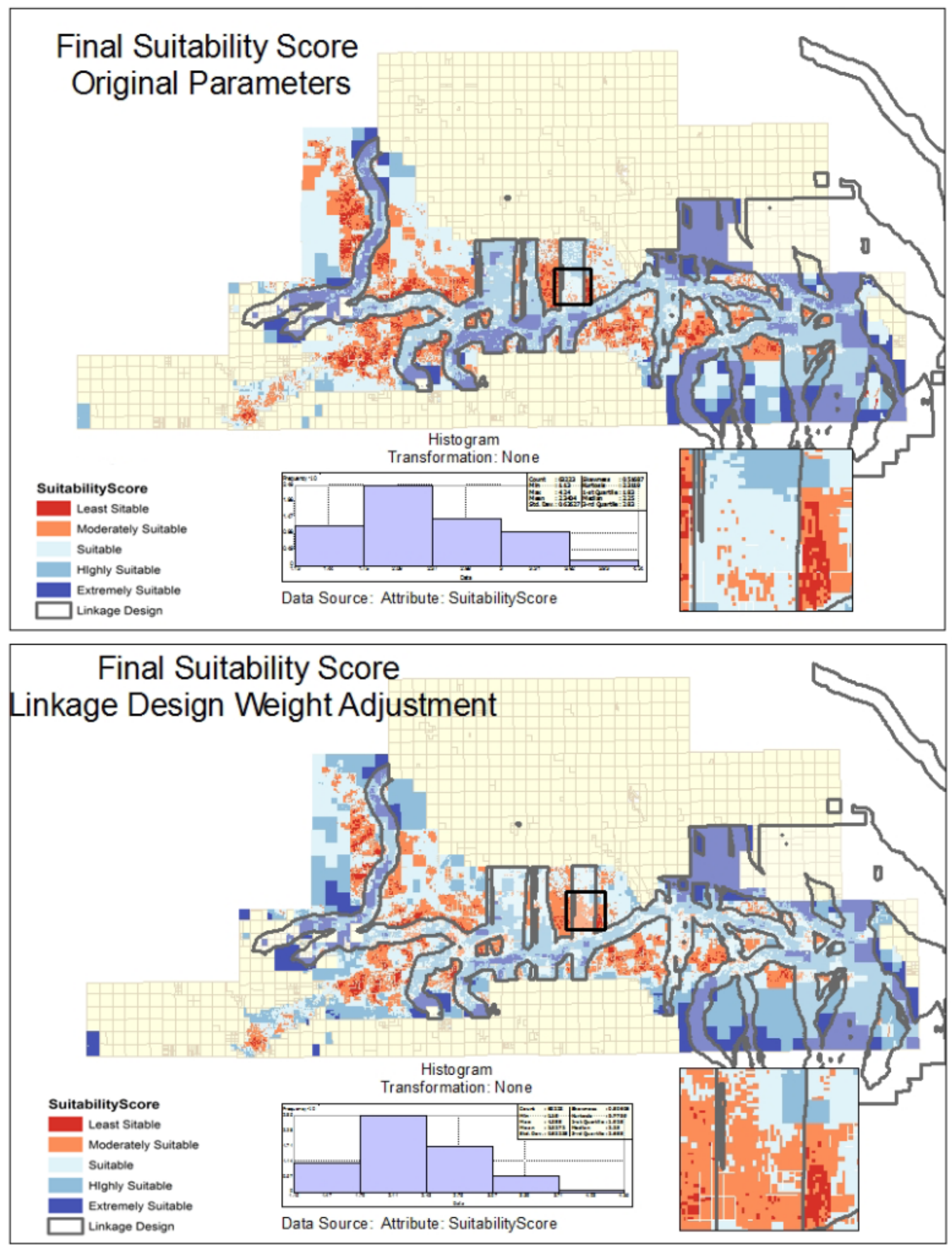

Figure 6-4: Linkage Design Weight Adjustment

A visual comparison of the two maps shows that areas within the linkage design that are developed (highlighted in the black box) received a lower score than in the original 
evaluation. This is important because these areas conflict with the goals of conservation and should be scoring lower. As expected, the most suitable areas still generally followed the linkage design. This is because the linkage design was created by an analysis of many factors that would reduce the permeability of the land.

If the user wanted to increase the appearance of areas for their greater opportunity for conservation they would reduce the weight of the factors that were taken into consideration in both this analysis and the SCW's linkage design creation, such as road density. Road density along with several other features such as slope, aspect, soils and vegetation were taken into consideration in the creation of the SCW linkage design. Road density was a large contributing factor in the permeability of the landscape for wildlife movement in general the linkage design should trace along areas with reduced road density there are several areas where this does not remain true and therefore road density was considered in this analysis. Therefore, to highlight areas that are more suitable based on conservation opportunity it is logical to reduce the impact of features previously considered in the SCW analysis such as road density. In contrast, they would increase the weight of the factors that are seen as indicative of greater conservation opportunity, such as nearness to conservation areas, parcel size, and vacancy (Table 6).

\section{Table 6. Opportunity Weight Adjustment Spreadsheet}

\begin{tabular}{|c|c|c|}
\hline Layer name & - Assigned Weight & - Percent Weight \\
\hline Planned for Development & Restricted & \\
\hline Currently Conserved & Restricted & \\
\hline Base Expansion Plans & Restricted & \\
\hline Vacant & & 21.4 \\
\hline \multirow[t]{2}{*}{ Linkage Design Interaction } & & 14.3 \\
\hline & & 0.0 \\
\hline Hydrography Density & & 7.1 \\
\hline Road Density & & 7.1 \\
\hline Parcel Size & & 21.4 \\
\hline Nearness to Conservation Areas & & 21.4 \\
\hline OHV Designated Routes & & 7.1 \\
\hline & & 14100 \\
\hline
\end{tabular}

In the example shown in Figure 6-8, the key factors that were increased to a weight of three were the nearness to conservation areas and parcel size. Vacancy was already weighted highly and did not need to be adjusted. The theory behind these factors being considered as indicative of greater conservation suitability is intuitive. The vacancy of the parcel is important because it is hard justify the conservation of a developed parcel over one that is undeveloped. Parcel size is important because parcels that are larger will conserve more land and could be used as stepping stone for a large conservation effort. 
Generally the idiom of greater bang for your buck applies here. The nearness to existing conservation areas is considered important because the goal of this conservation effort is not to protect islands of habitat but to protect land that will connect the existing conservation areas. For this reason, conserving a parcel that is closer to an existing conservation area is more justifiable than conserving one that is far from one. The effects of these shifts were mapped and symbolized to visualize the changes in pattern that occurred because of this adjustment (Figure 6-5). 

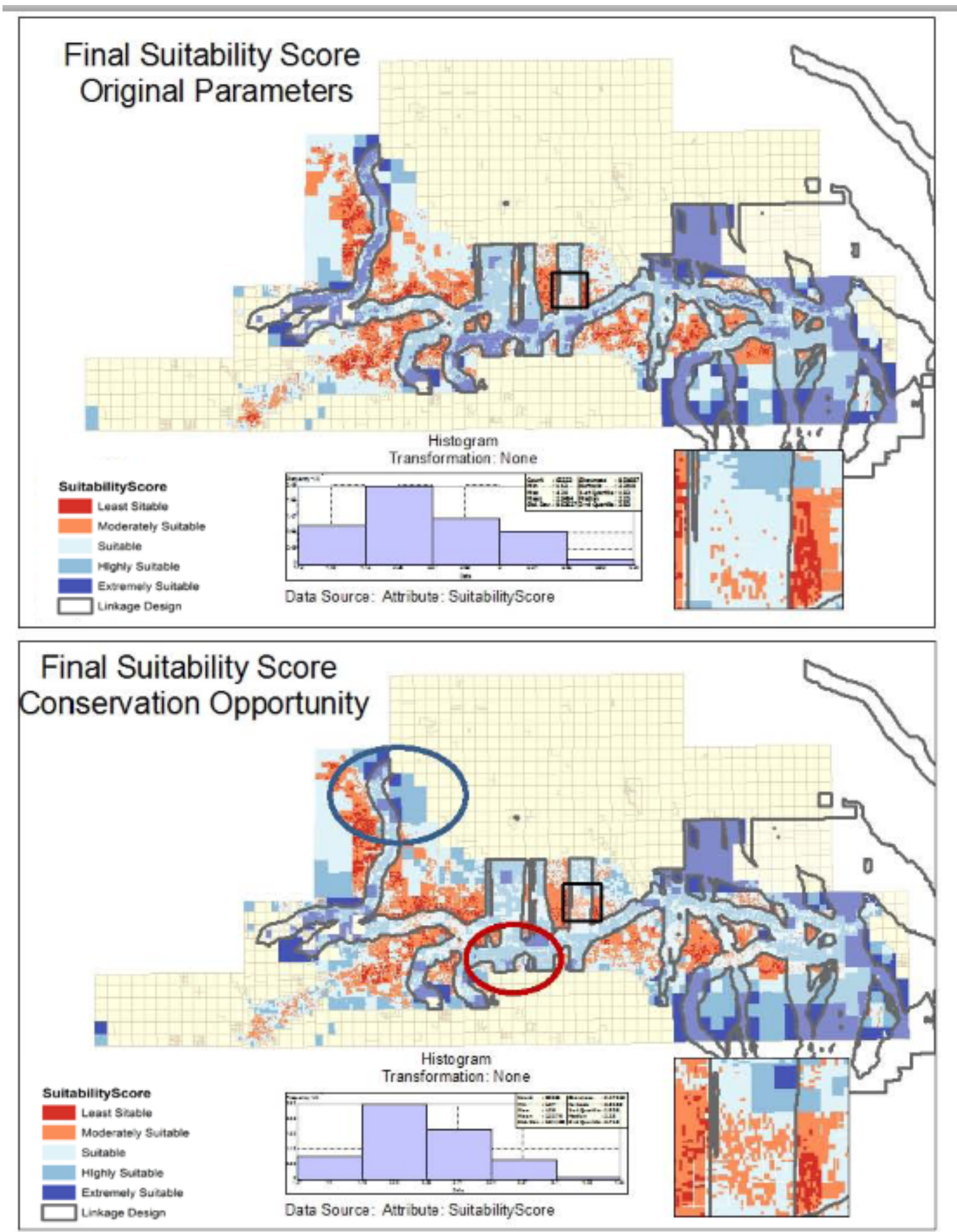

\section{Figure 6-5: Conservation Suitability Weight Adjustment}

The areas that were close to existing conservation areas increased in suitability score (highlighted with a blue circle). However, there are a few areas whose score decreased 
even though they are close to a conservation area (highlighted with a red circle). This is most likely because the parcel was small and not vacant. In addition, the parcels within the linkage design area are generally become less suitable, simply because the linkage design factor become less important in this example.

The final example describes a situation where in the user wanted to consider all factors equally. This could be used to set a baseline of the impact of each of the factor. With all the factors being equally weighted, the user can visualize the simple interaction of the information without weighting (Table 7).

\section{Table 7. Equal Weight Adjustment Spreadsheet}

\begin{tabular}{|c|c|c|}
\hline Layer name & Assigned Weight & Percent Weight \\
\hline Planned for Development & Restricted & \\
\hline Currently Conserved & Restricted & \\
\hline Base Expansion Plans & Restricted & \\
\hline Vacant & 1 & 14.29 \\
\hline \multirow[t]{2}{*}{ Linkage Design Interaction } & 1 & 14.29 \\
\hline & & 0.00 \\
\hline Hydrography Density & 1 & 14.29 \\
\hline Road Density & 1 & 14.29 \\
\hline Parcel Size & 1 & 14.29 \\
\hline Nearness to Conservation Areas & 1 & 14.29 \\
\hline \multirow[t]{2}{*}{ OHV Designated Routes } & 1 & 14.29 \\
\hline & 7 & 100 \\
\hline
\end{tabular}

The equal weights were entered into the model and the model was rerun. The results were mapped and symbolized to better visualize the effects each of those weights had on the pattern of the suitability score (Figure 6-6) 

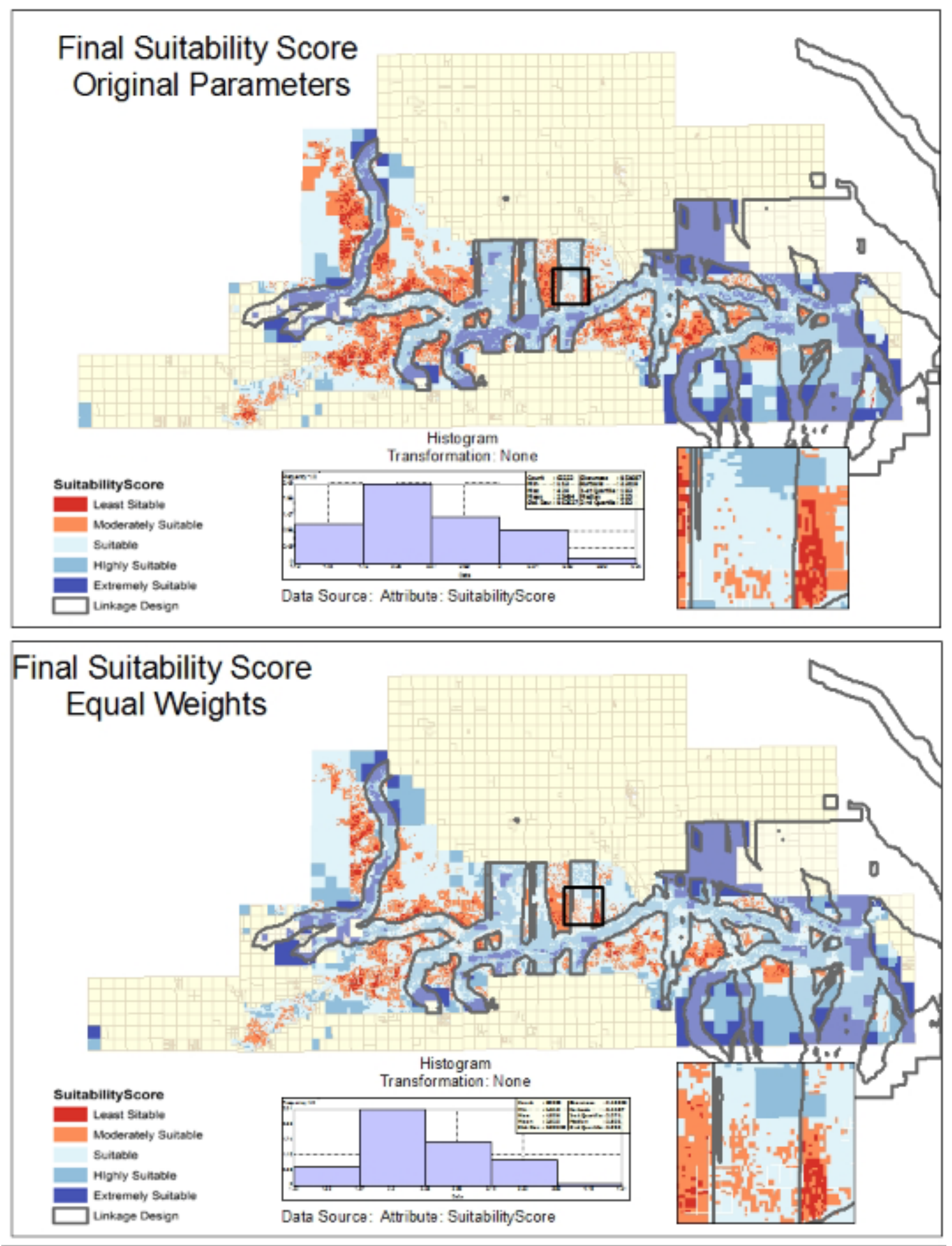

Figure 6-6: Equal Weights Analysis 
Surprisingly, the change in pattern was not significant. This could be explained because some of the features negate another's impact. For example, the effects of the OHV-designated areas criterion are diminished because the parcels are also considered vacant they also tend to be closer to existing conservation areas. Many of these factors act synergistically and that should be taken in to consideration when weighting these features. The weights can either reduce or increase this effect and that effect should be taken into consideration.

\subsubsection{Adjusting Excluded Features}

In the original analysis, there were three factors used to exclude parcels for suitability analysis as these are restricting factors for conservation: parcels planned for development, parcels currently conserved and parcels planned for base expansion. In practice, the end user can also choose exclusive factors to meet their needs. An example of adjusting the excluded features in the analysis could be the exclusion of all parcels that do not interact with the linkage design from evaluation. If the user wanted to evaluate only the parcels within the linkage design, the weight of the linkage design will be eliminated. The suitability analysis results for this situation are displayed in Figure 6-7. 


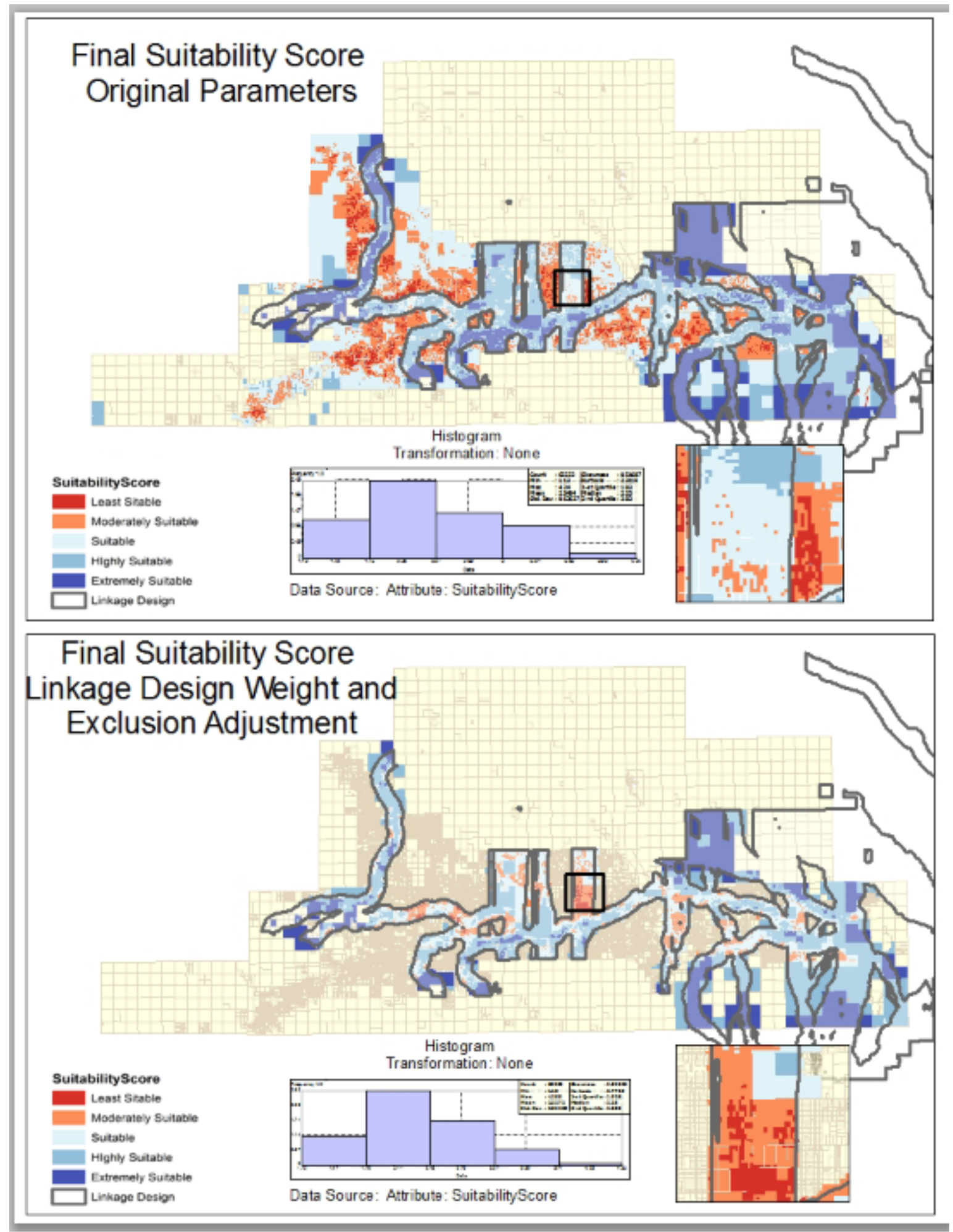

Figure 6-7: Exclusive Linkage Design Suitability Analysis 
This analysis is very helpful because it not only identifies areas that are highly suitable but also identifies areas that highly conflict with the effort of conservation and should be considered for stewardship efforts instead.

\subsection{Application of Suitability Results}

After suitability scores are calculated, the parcels in the basin can be analyzed in a variety of ways. The suitability of the scores can be queried and their relationship with various features in the basin can be evaluated. The analysis of the parcels and their suitability for conservation is the job of the end user. However, for the purposes of this project, several sample analysis were done to demonstrate potential analyses, such as: stewardship analysis, city zoning interactions, and BLM undesignated areas analysis.

\subsubsection{Stewardship Analysis}

This analysis was done by selecting only the parcels that interact with the linkage design and creating a layer from them. The layer was created to decrease the processing time for the analysis. Once the layer was created, a hotspot analysis was done. The hotspot analysis was run using the Hot Spot Analysis (Getis Ord Gi*) spatial statistics tool in arc toolbox. The areas in blue show a clustering of parcels that have lower suitability scores. These areas are most likely scoring lower because they are not vacant, have high density of roads and the parcels are small (Figure 6-8). 


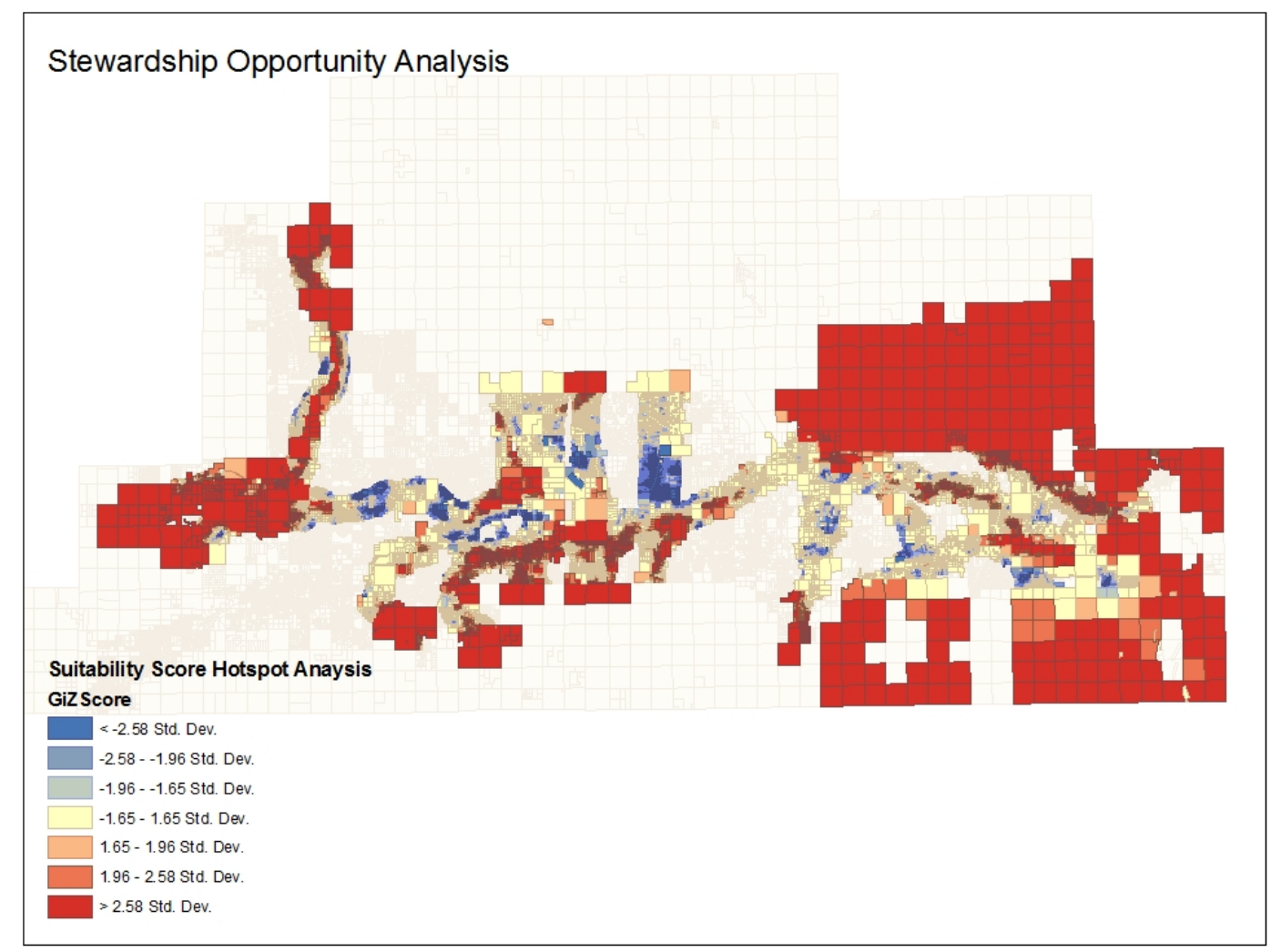

\section{Figure 6-8: Exclusive Linkage Design Suitability Analysis}

These areas are most likely communities and could be considered by a planner as areas that should be targeted for stewardship efforts. Stewardship has been recommended by the SCW and is a goal of the MBOSG. For this reason, analysis such as this can be very helpful in concentrating those efforts. Examples of good stewardship include reducing speed limits, reducing or eliminating the use of herbicides and pesticides, keeping predatory pets indoors, and landscaping with native plants. Awareness of these practices would have to be cultivated in these communities and a targeted effort would greatly reduce expenses and increase effectiveness.

\subsubsection{City Zoning Analysis}

The land suitability scores can also be integrated with other data layers to conduct other analysis. For example, it can be overlaid with city zoning to examine the interaction between the land suitability for conservation and city zoning policies. Specifically, using the city zoning, the end user could determine which parcels are most suitable for conservation without conflicting with the city zoning. One such city zoning type that would be compatible with conservation is the Rural Living Zone. The Wildlands Conservancy (TWC) has already conserved several parcels that have been zone for rural living. Areas that have high conservation suitability can be mapped and overlaid with the 
city zoning to visualize their interaction. The city zoning is very complex and when mapped the rural living zoning area, symbolized with a dark blue boundary line, begin to coalesce this is seen in Figure 6-9.

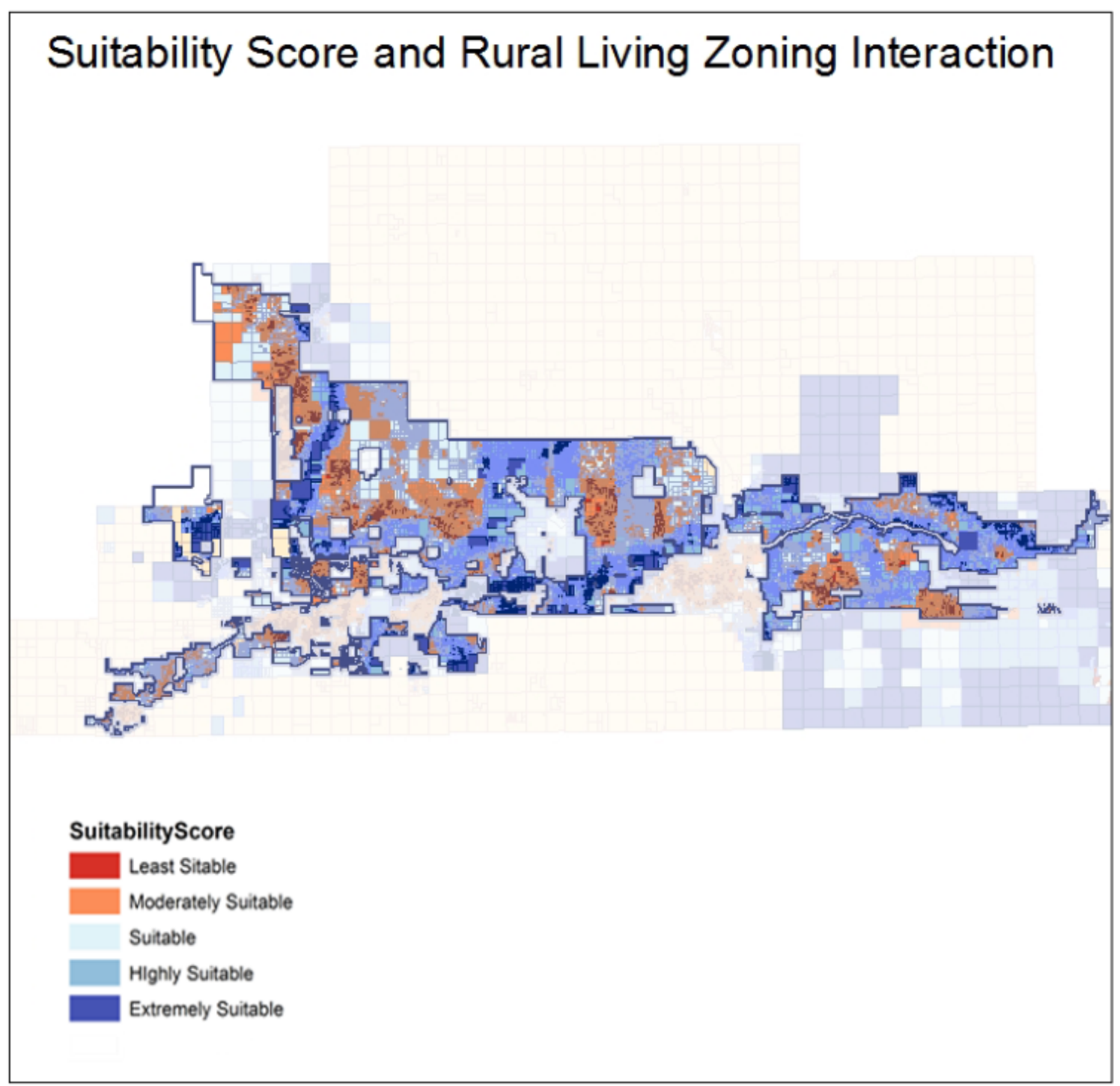

Figure 6-9: Rural Living and Suitability Score Analysis

In order to visually interpret the interaction of these layers, the user would have to zoom into focus areas. However, the analysis could be done by querying the attribute table for parcels that have a high suitability score and are zoned for rural living. The APN of the parcels that fall into the rural living code can be obtained by intersecting the parcel dataset with the rural living data. The results of this intersection can be used by the end user or planner to select only those parcels that are both highly suitable and do not conflict with the zoning in the area. 


\subsubsection{Suitability of Unclassified BLM land}

The final analysis that was demonstrated was of undesignated BLM lands and their relative suitability for conservation. Undesignated BLM land has the potential to be used for multiple purposes that may not be conducive to wildlife movement. One of these conflicting uses is the development of renewable energy sites. Using the exclusion criteria, the user can exclude all areas that are not undesignated BLM land, the end user can identify the undesignated BLM parcels that are most suitable for conservation and identify those that could be used for renewable energy (those with the least suitability scores) (Figure 6-10).

\section{Undesignated BLM Land Suitability Score}

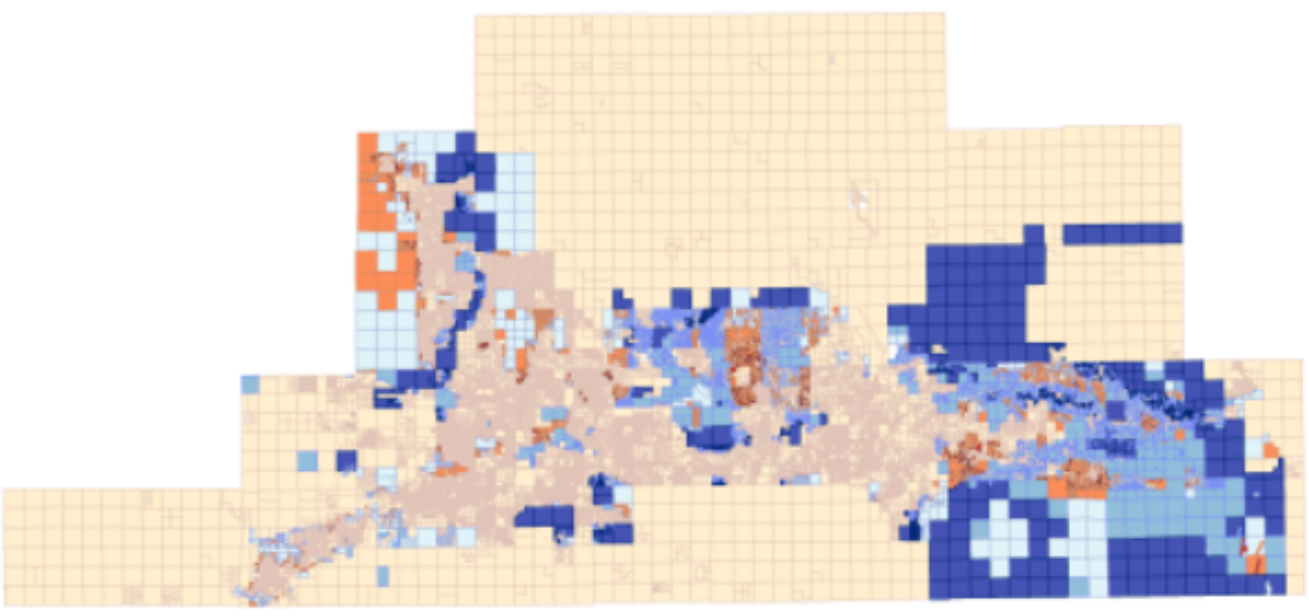

Final Suitability Score of BLM

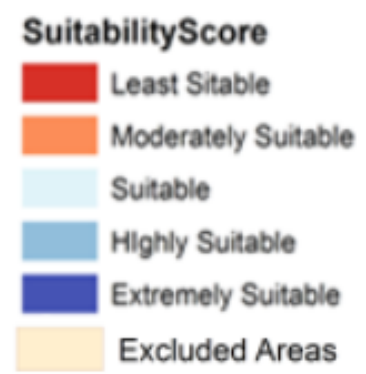

Figure 6-10: Undesignated BLM Land Suitability Analysis

With the results from this analysis, the end user could make the case to the BLM for the designation of some BLM areas as protected and allow multiple uses to continue on other areas. 


\subsection{Summary}

The suitability score that was produced by the model was as expected. The modifiability of the model was essential in tuning to meet the goals of individual analyses. Shifting the criteria classifications or weights will have some effect on the suitability score received for each parcel. It has been determined that several factors interact synergistically in the scoring need to be considered in the setting of the weights. In addition, with the use of some of the tools in ArcMap the suitability score can be used to identify hotspots for stewardship and analyze the interaction of the land suitability for conservation with other important datasets, such as zoning. In doing so, more comprehensive conservation plan can be made without conflicting with the current planning policies. 



\section{Chapter 7 - Conclusions and Future Work}

\subsection{Conclusion}

This project was designed to meet the needs of the client and the MBOSG. They wanted a repeatable and modifiable model that would prioritize parcels in the basin for conservation based on specific criteria. As a result, this suitability analysis and model produces a single suitability score that can be analyzed to develop an action plan for conservation.

The client wanted a model that was easy to use, modify, and update. The model that was developed was easy to use due the use of Python scripting to automate the modification process. The model was modifiable through a geodatabase table where the user could adjust the criteria classification breaks and weights. All of the data needed in the development of the model were stored in an associated geodatabase and ready for use in future projects. The update process for the model was not automated. Any updates to the model would have to be done within the project dataset. These features were not incorporated into the model because of time constraints. The addition if these capabilities should be considered for future work. The model did, even without the ability to update the data, meet the critical needs set by the client and this project was considered a success.

The results were simple, quantifiable, and capable of being queried. They can be used to identify areas that conflict with the goals of wildlife movement, can be analyzed for stewardship, and can be queried to examine the suitability of only vacant parcels or BLM undesignated areas.

\subsection{Potential Extensions}

\subsubsection{Threats vs. Opportunity Analysis}

The model developed in this project considered only features that were indicative of opportunity and did not incorporate the potential threats of development to the parcel. Across the basin there are areas that are more suitable for development than others. Areas that are not suitable for development most likely will not be developed in the near future and should not be set as a priority for conservation. Rather parcels that have a high opportunity and value for conservation but also are highly suitable for development should be prioritized for conservation. The threats element of evaluation was not incorporated into this evaluation because of limited data availability and time constraints. In order to incorporate this into the model a basin wide development suitability analysis would have to be completed and those results incorporated appropriately into the model. 


\subsubsection{Species Specific Analysis}

The SCW did their analysis using specific species. This data, which was not available at the time of this project's planning, could be incorporated into the prioritization model and the end user could prioritize areas based on the parcel's suitability for conservation but also its propensity to support a key species; for example, desert tortoise or big horn sheep. With this extension, the interface could be modified so the end user could easily indicate which species are to be considered, what landscape features should be included and excluded from analysis, and how they should be weighted. This would require a custom interface for the application and would replace the existing interface.

\subsubsection{Model Corridor Loss Effects}

Another useful addition to the analysis would be to model the effects of extensive development with an identified potential corridor area. In order to complete this, the developer would have to obtain the species specific information that was used by the $\mathrm{SCW}$ in the development of their linkage design. Using this data, the developer would then have to create a model that would simulate the existence of features that negatively affect the permeability of the land for wildlife movement. These simulations could be randomly calculated or placed based on a development suitability score of the land. The goal of this would be to show areas that are irreplaceable because their loss would mean a significant decline in the mobility potential of the focus species. This information would aide in the development of priority efforts, as well as the development of mitigation plans.

\subsubsection{Develop a General Conservation Prioritization Template and Toolset}

The SCW has developed a series of linkage designs across the state and is currently underway to create 22 linkage designs to stitch together the Mojave Desert conservation areas. The application of a prioritization model that could be applied to each of those linkage designs would greatly enhance the efficiency of the land managers interested in implementing these designs. This would require the creation of a toolset that would automate the preprocessing of the datasets and generic model that could be applied to any region. In addition, a template geodatabase design would be created to store necessary data. Guidelines for data quality and scale would have to be determined and an easy-touse interface to be created. 


\section{Works Cited}

AL_SHALABI, M., Mansor, S., Ahmed, N., \& Shariff. (2006). GIS Based Multicriteria Approaches to Housing Site Siutability Assessment. Munich: Shaping Change .

Clevenger, A. P., Wierzchowski, J., Chruszcz, B., \& Gunson, K. (2002). GIS-Generated, Expert-Based Models for Identifying Wildlife Habitat Linkages and Planning Mitigation Passages. Conservation Biology, 503-514.

Debinski, D. M., \& Holt, R. D. (2000). A Survey and Overview of Habitat Fragmentation Experiments. Conservation Biology, 342-355.

Forman, R. T., \& Deblinger, R. D. (2000). The Ecological Road-Effect Zone of a Massachusetts (U.S.A.) Suburban Highway. Conservation Biology, 36-46.

Gilpin, M. E., \& Soule, M. E. (1986). Minimum viable populations: processes of species extinction. Conservation biology: the science of scarcity and diversity , 19-34.

Haddad, N. M. (1999). Corridor and distance effects on interpatch movements: a landscape experiment with butterflies. Ecological Applications , 612-622.

Haddad, N. M., \& Baum., K. A. (1999). An experimental test of corridor effects on butterfly densities. Ecological Applications , 623-633.

Hall, G., Wang, F., \& Subaryono. (1992). Comparison of Boolean and fuzzy classification methods in land suitability analysis by using geographical information systems . Environment and Planning , 497-516.

Jankowski, P. (1995). Intergrating geographical information systems and multiple criteria decisions-making methods. International Journal of Geographical Information Systems , 251-273.

Joerine, F., Theriault, M., \& Musy, A. (2001). Using GIS and outranking multicriteria analysis for land-use suitability assessment . International Journal of Geographical Information Systems , 153-174.

L. S. Hall, M. K. (2000). Spatial organization and habitat use of feral cats (Felis catus L.) in Mediterranean California. Mammalia , 19-28.

Mader, H. (1984). Animal habitat isolation by roads and agricultural feilds,. Biological Conservation, 81-96.

Marzluff, J. M., \& Ewing, K. (2001). Restoration of fragmented landscapes for the conservation of birds: A general framework and specific recommendations for urbanizing landscapes. Restoration Ecology, 280-292.

Pacific Biodiversity Institute. (2002). Natural Rescource Information and Conservation Decision Support for the Wenatchee River Basin. Winthrop, WA: Pacific Biodiversity Institute.

Penrod, K., Cabañero, C., Beier, P., Luke, C., Spencer, W., Rubin, E., et al. (2008). A Linkage Design for the Joshua Tree-Twentynine Palms Connection. Fair Oaks, CA: South.

Schmiegelow., F. K., Machtans, C. S., \& Hannon., S. J. (1997). Are boreal birds resilient to forest fragmentation: an experimental study of short-term community responses. Ecology, 1914-1932. 
Shultz, K., Larsen, J., Cunningham, L., Forsyth, M., \& Toft, T. (2003). Coastal Mississippi Land Development Suitability Model. ESRI International User Conference.

Smith, D. (1999). Identification and prioritization of ecological interface zones on state highways in Florida. Proceedings of the third international conference on wildlife ecology and transportation (pp. 209-230). Florida Department of Transportation,: Florida Department of Transportation.

South Coast Wildlands. (2008). South Coast Missing Linkages: A Wildland Network for the South Coast Region. Fair Oaks, CA: South Coast Wildlands.

Steiner, F., \& McSherry, L. (2000). Land suitability analysis for the upper Gila River watershed. Landscape and Urban Planning , 50, 199 -214.

Theobald, D. (2002). Modeling Functional Landscape Connectivity. Proceedings of the Twenty-Second Annual ESRI User Conference. San Diego, CA: ESRI.

Treweek, J., \& Veitch., N. (1996). The potential application of GIS and remotely sensed data to the ecological assessment of proposed new road schemes. Global Ecology and Biogeography Letters , 249-257.

Wang, F. (1994). The use of artificaial nueral networks ina geographical information systems for agriculture land-suitability assessment. Environment and Planning, 265-284.

Wolff, J. O., Schauber, E. M., \& Edge., W. D. (1997). Effects of habitat loss and fragmentation on the behavior and demography of graytailed voles. Conservation Biology, 945-956. 


\section{Appendix A. Density Line Density Calculation Python Script}

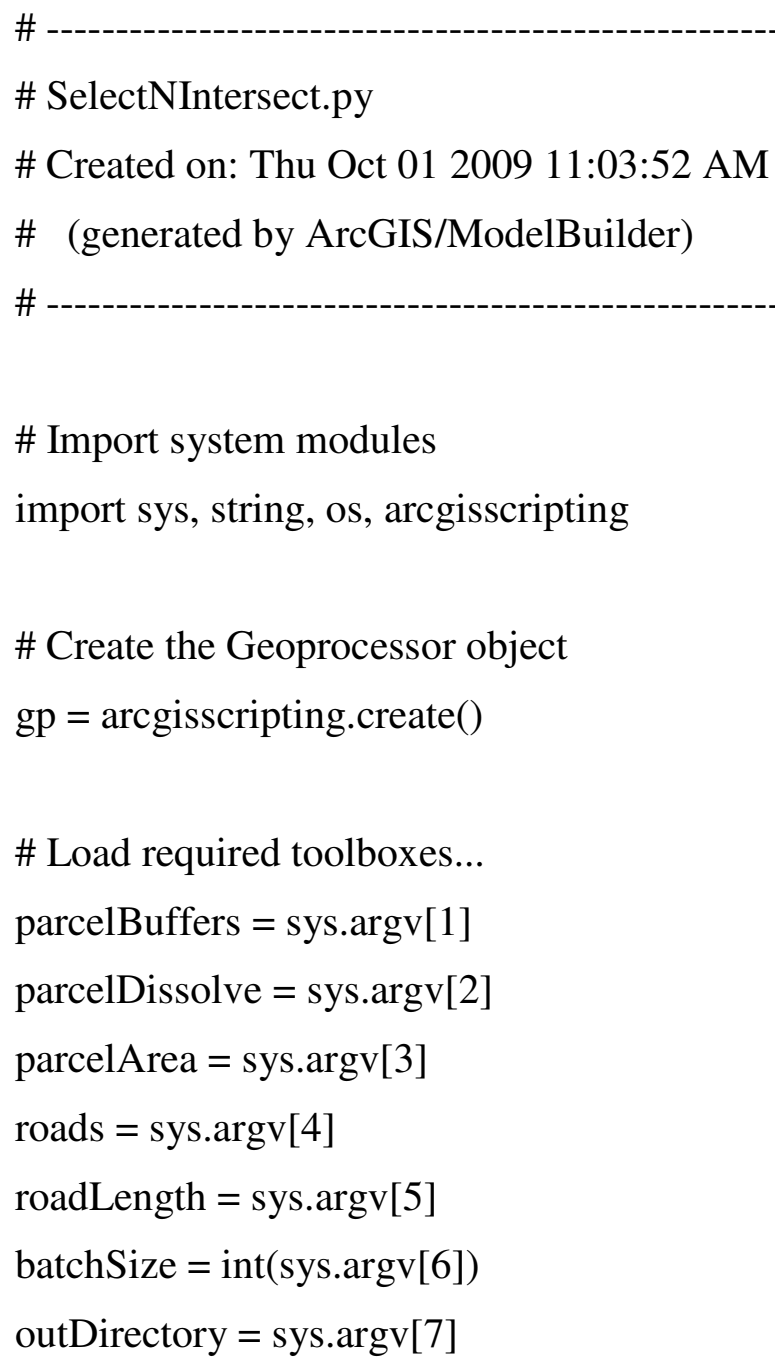




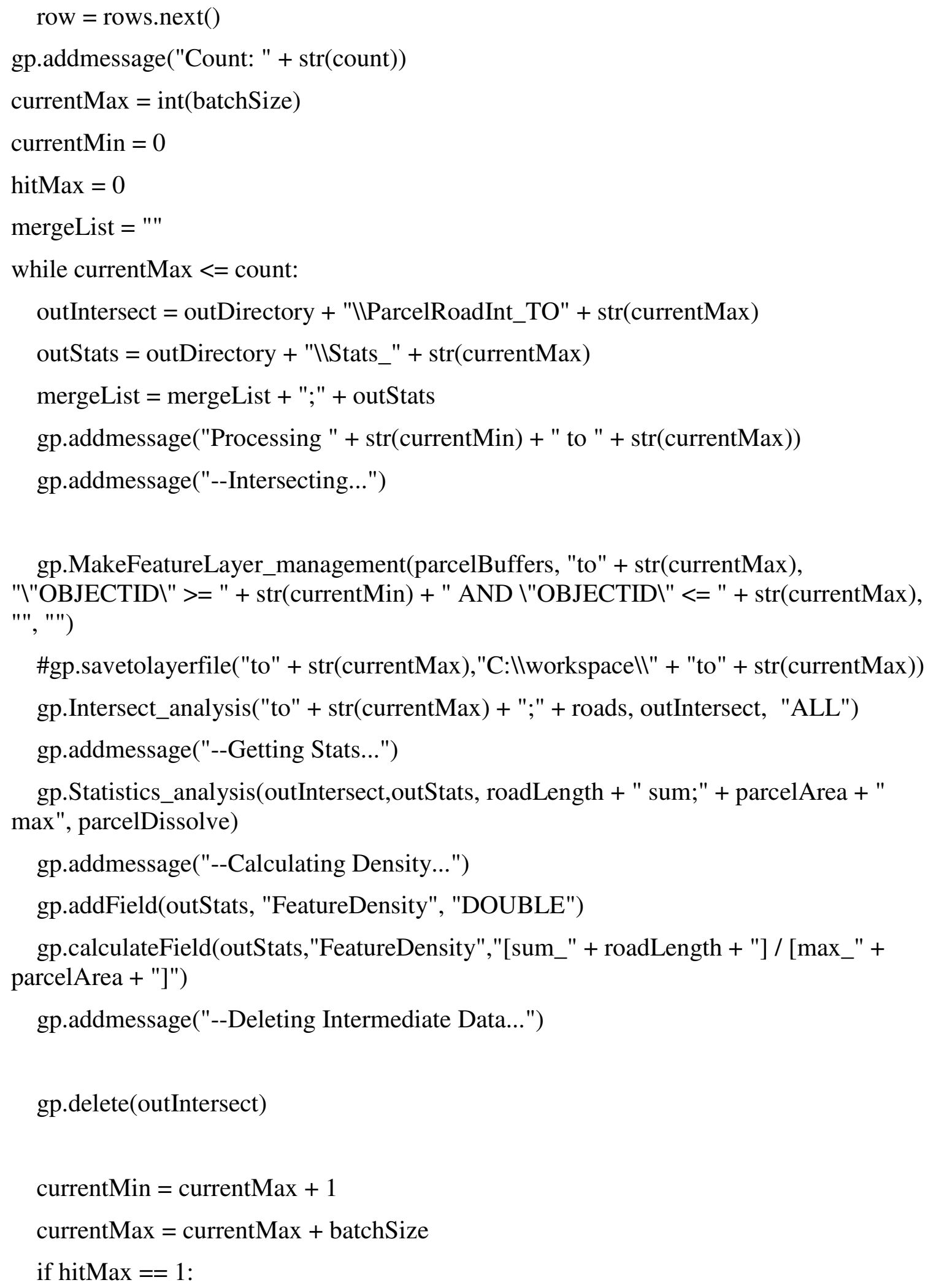




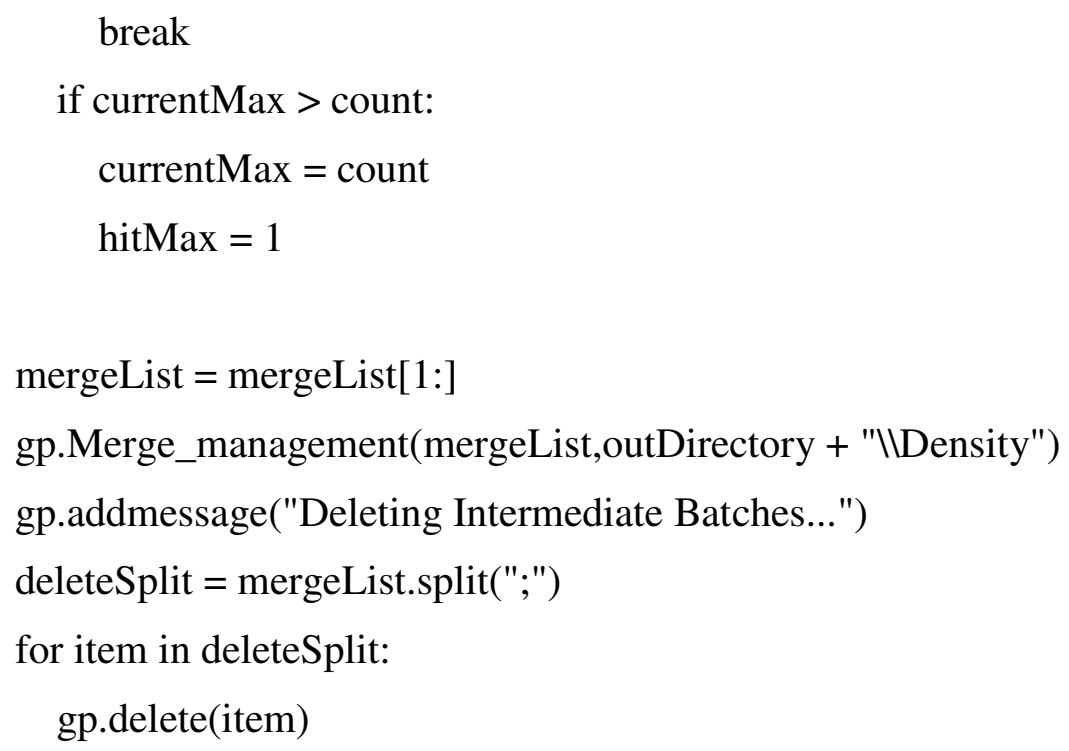

gp.addmessage(" Density Operation Complete. Final data at: " + outDirectory + "IDensity") 


\section{Appendix B. ModelBuilder Diagrams}

ModelBuilder tool that adds the reclassification fields to the parcel dataset. 


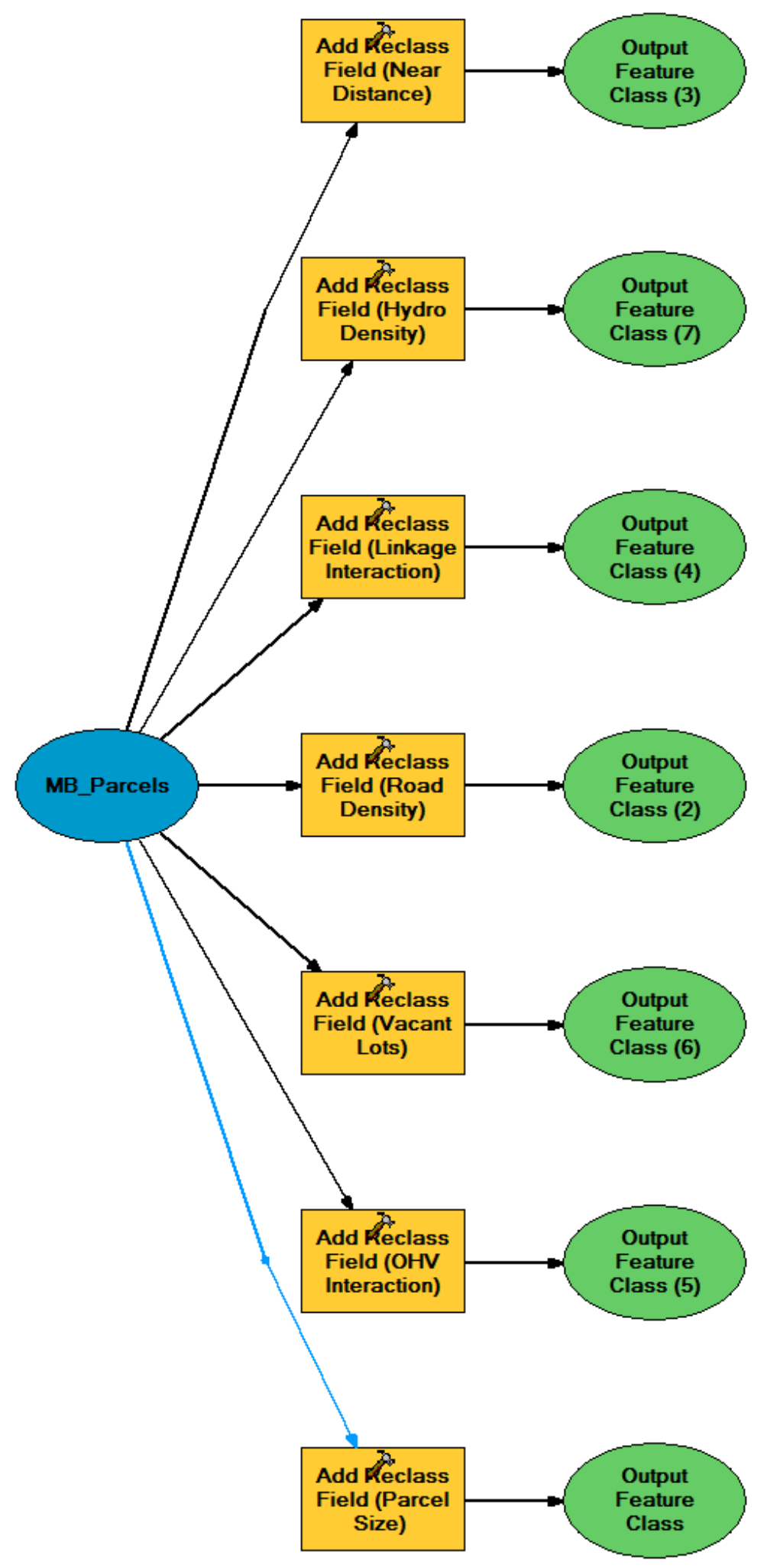


This ModelBuilder tool calculates and adds the weight field and factor suitability scores. This image has been cut in half then enlarged to allow for better legibility (Diagram A and B).

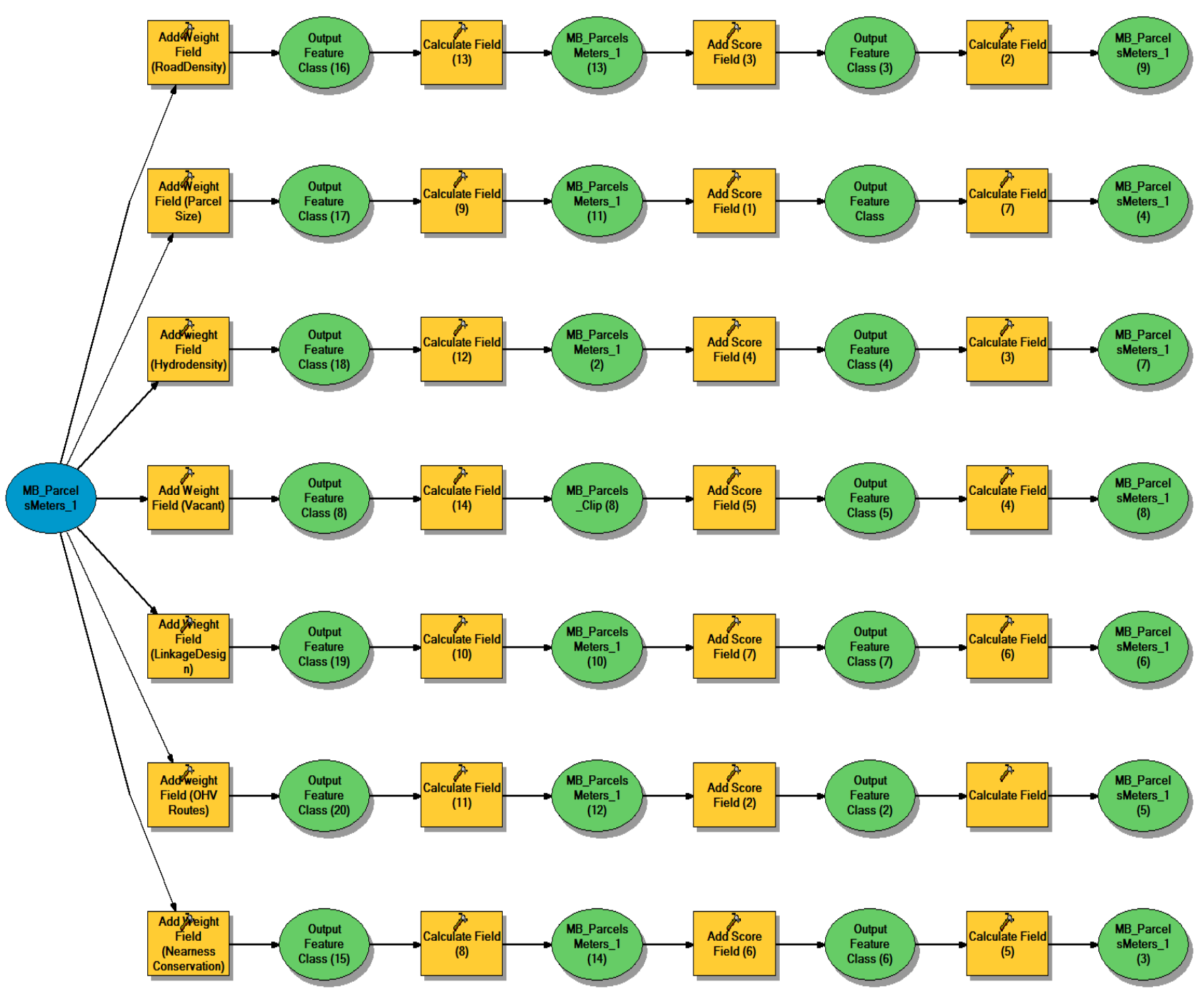

\section{Complete Diagram}




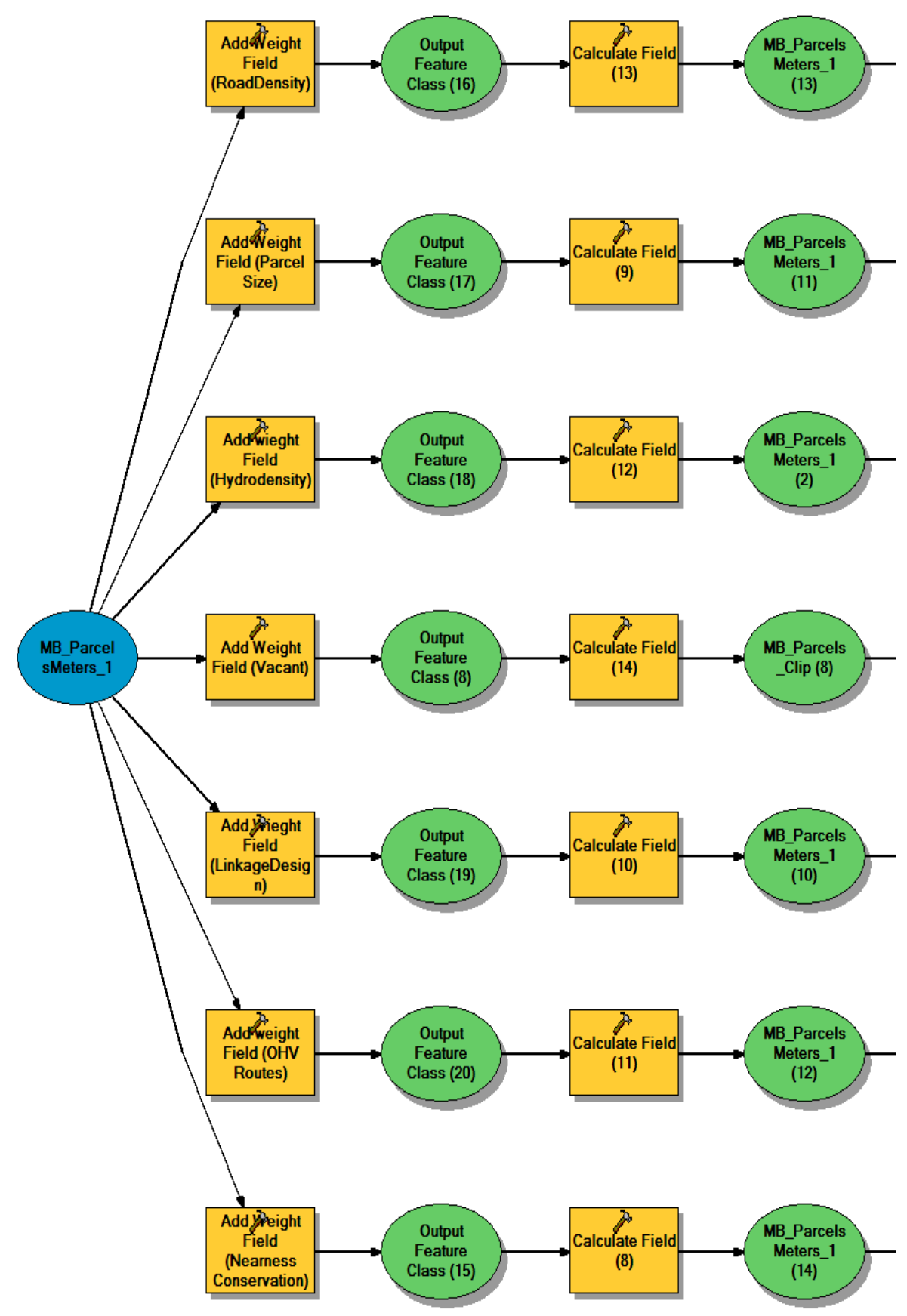

\section{Diagram Part A}



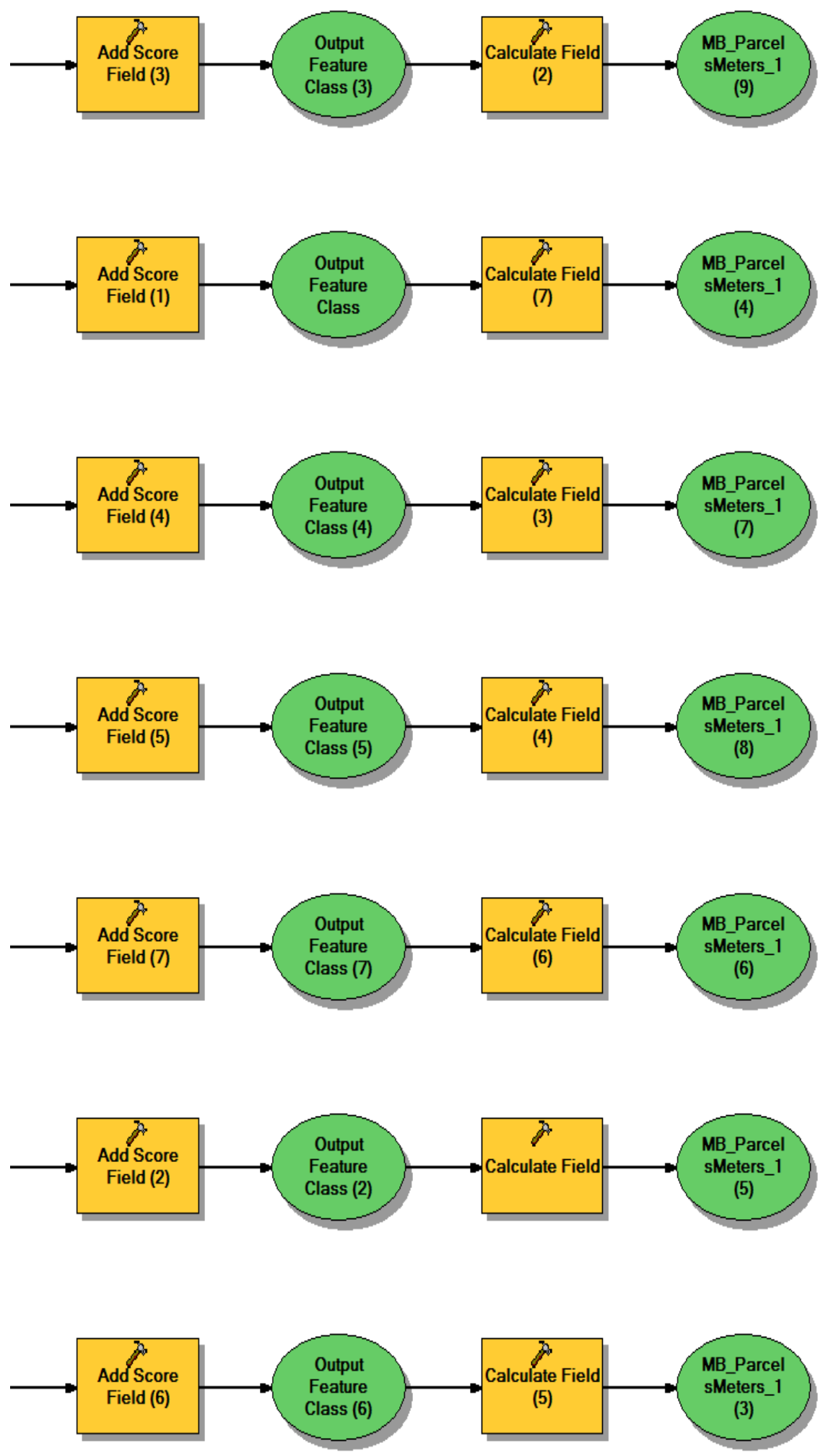

\section{Diagram Part B}




\section{Appendix C. Suitability Score Calculation Python Script}

"'

Created on Nov 6, 2009

@ author: nathan_strout

"'

import arcgisscripting

import os, sys

$\mathrm{gp}=$ arcgisscripting.create $(9.3)$

\#Get parameters from tool

inFeatureClass $=$ gp.GetParameter $(0)$

inFactorClassTable $=$ gp.GetParameter $(1)$

outFeatureClass = gp.GetParameter(2)

\#inFeatureClass =

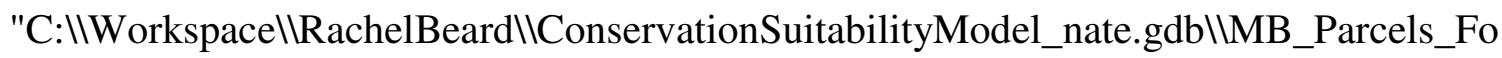
cusData"

\#inFactorClassTable $=$

"C:॥Workspacel|RachelBeard \ConservationSuitabilityModel_nate.gdb\\CriteriaBreaks"

\#outFeatureClass =

"C:॥Workspacel\RachelBeard \ConservationSuitabilityModel_nate.gdb\\NateReclass"

\#gp.overwriteresults = True

gp.addMessage("Creating Output...")

gp.copy(inFeatureClass,outFeatureClass)

\#Verify Weights

rows $=$ gp.SearchCursor(inFactorClassTable $)$

row $=$ rows.next ()

weightTotal $=0.0$

while row:

\#gp.addmessage(str(row.GetValue("Weight"))) 


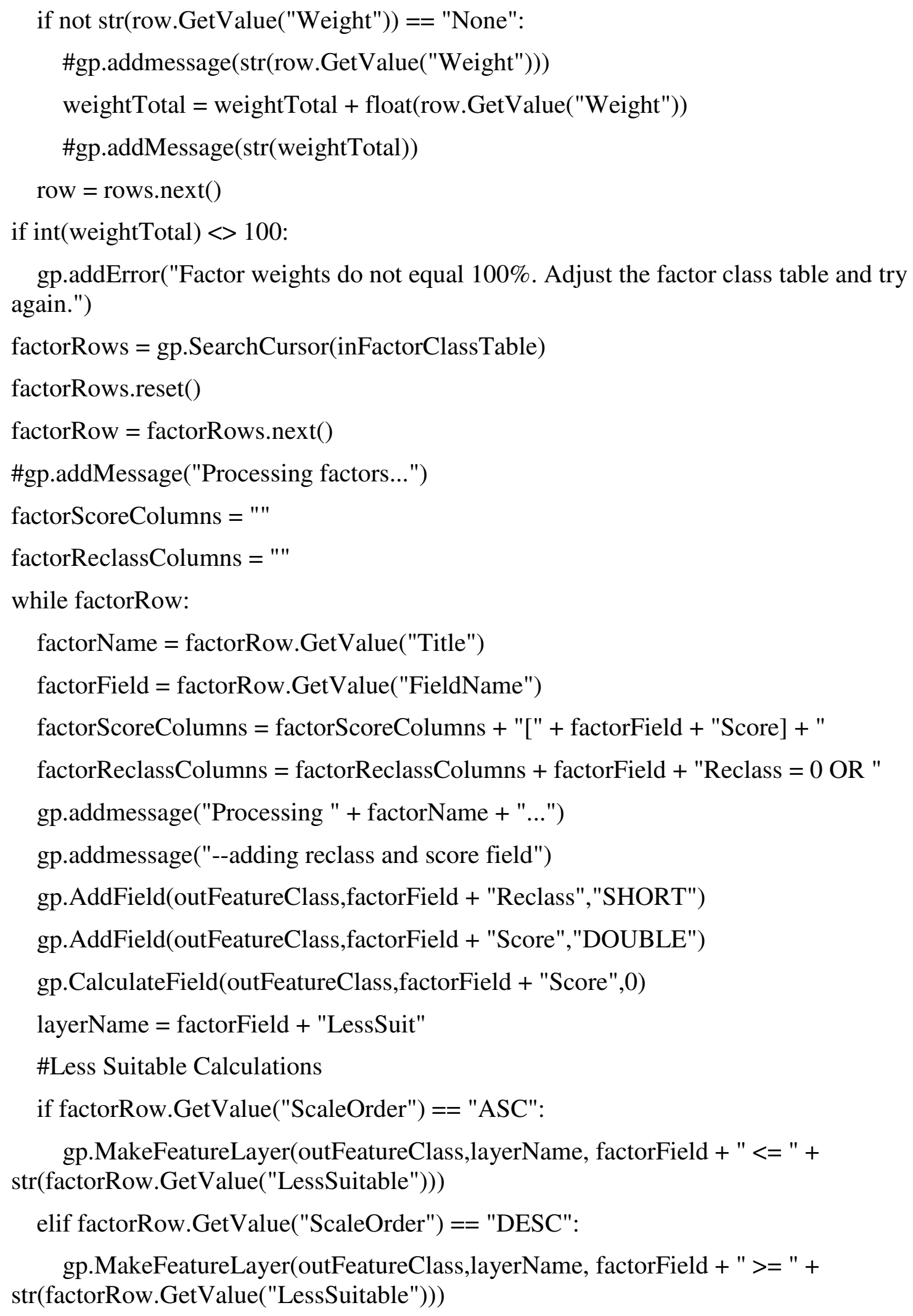


elif factorRow.GetValue("ScaleOrder") == "BOOL":

gp.MakeFeatureLayer(outFeatureClass,layerName, factorField + " = " + str(factorRow.GetValue("LessSuitable")))

gp.addmessage("--Calculating low suitable values")

gp.CalculateField(layerName,factorField + "Reclass", 1)

if not str(factorRow.GetValue("Weight")) == "None":

gp.CalculateField(layerName,factorField + "Score", "[" + factorField + "Reclass] * " + str(float(factorRow.GetValue("Weight")) / 100))

\#Moderate Suitable Calculations

if factorRow.GetValue("ScaleOrder") == "ASC":

gp.MakeFeatureLayer(outFeatureClass,layerName, factorField $+"<="+$ str(factorRow.GetValue("ModerateSuitable")) + " AND " + factorField + " > " + str(factorRow.GetValue("LessSuitable")))

elif factorRow.GetValue("ScaleOrder") == "DESC":

gp.MakeFeatureLayer(outFeatureClass,layerName, factorField + " >= " + str(factorRow.GetValue("ModerateSuitable")) + " AND " + factorField + " < " + str(factorRow.GetValue("LessSuitable")))

elif factorRow.GetValue("ScaleOrder") == "BOOL":

gp.MakeFeatureLayer(outFeatureClass,layerName, factorField + " = " + str(factorRow.GetValue("ModerateSuitable")))

gp.addmessage("--Calculating moderate suitable values")

gp.CalculateField(layerName,factorField + "Reclass", 2)

if not str(factorRow.GetValue("Weight")) == "None":

gp.CalculateField(layerName,factorField + "Score", "[" + factorField + "Reclass] * " $+\operatorname{str}($ float(factorRow.GetValue("Weight")) / 100))

\#Suitable Calculations

if factorRow.GetValue("ScaleOrder") == "ASC":

gp.MakeFeatureLayer(outFeatureClass,layerName, factorField $+"<="+$ str(factorRow.GetValue("Suitable")) + " AND " + factorField + " > " + str(factorRow.GetValue("ModerateSuitable")))

elif factorRow.GetValue("ScaleOrder") == "DESC":

gp.MakeFeatureLayer(outFeatureClass,layerName, factorField + " >= " + str(factorRow.GetValue("Suitable")) + " AND " + factorField + " < " + str(factorRow.GetValue("ModerateSuitable")))

elif factorRow.GetValue("ScaleOrder") == "BOOL": 
gp.MakeFeatureLayer(outFeatureClass,layerName, factorField + " = " + str(factorRow.GetValue("Suitable")))

gp.addmessage("--Calculating suitable values")

gp.CalculateField(layerName,factorField + "Reclass", 3)

if not str(factorRow.GetValue("Weight")) == "None":

gp.CalculateField(layerName,factorField + "Score", "[" + factorField + "Reclass] * " + str(float(factorRow.GetValue("Weight")) / 100))

\#High Suitable Calculations

if factorRow.GetValue("ScaleOrder") == "ASC":

gp.MakeFeatureLayer(outFeatureClass, layerName, factorField + " $<="+$ str(factorRow.GetValue("HighSuitable")) + " AND " + factorField + " > " + str(factorRow.GetValue("Suitable")))

elif factorRow.GetValue("ScaleOrder") == "DESC":

gp.MakeFeatureLayer(outFeatureClass,layerName, factorField + " >= " + str(factorRow.GetValue("HighSuitable")) + " AND " + factorField + " < " + str(factorRow.GetValue("Suitable")))

elif factorRow.GetValue("ScaleOrder") == "BOOL":

gp.MakeFeatureLayer(outFeatureClass,layerName, factorField + " = " + str(factorRow.GetValue("HighSuitable")))

gp.addmessage("--Calculating high suitable values")

gp.CalculateField(layerName,factorField + "Reclass", 4)

if not str(factorRow.GetValue("Weight")) == "None":

gp.CalculateField(layerName,factorField + "Score", "[" + factorField + "Reclass] * " + str(float(factorRow.GetValue("Weight")) / 100))

\section{\#Extremely Suitable Calculations}

if factorRow.GetValue("ScaleOrder") == "ASC":

gp.MakeFeatureLayer(outFeatureClass,layerName, factorField + " > " + str(factorRow.GetValue("HighSuitable")))

elif factorRow.GetValue("ScaleOrder") == "DESC":

gp.MakeFeatureLayer(outFeatureClass,layerName, factorField + " < " + str(factorRow.GetValue("HighSuitable")))

elif factorRow.GetValue("ScaleOrder") == "BOOL":

gp.MakeFeatureLayer(outFeatureClass,layerName, factorField + " = " + str(factorRow.GetValue("HighSuitable"))) 
gp.addmessage("--Calculating extremely suitable values")

gp.CalculateField(layerName,factorField + "Reclass", 5)

if not $\operatorname{str}($ factorRow.GetValue("Weight")) == "None":

gp.CalculateField(layerName,factorField + "Score", "[" + factorField + "Reclass] * " + str(float(factorRow.GetValue("Weight")) / 100))

\#Unsuitable Calculations

gp.MakeFeatureLayer(outFeatureClass,layerName, factorField + " = " + str(factorRow.GetValue("Unsuitable")))

gp.addmessage("--Calculating unsuitable values")

gp.CalculateField(layerName,factorField + "Reclass", 0)

if not str(factorRow.GetValue("Weight")) == "None":

gp.CalculateField(layerName,factorField + "Score", "[" + factorField + "Reclass] * " + str(float(factorRow.GetValue("Weight")) / 100))

factorRow $=$ factorRows.next ()

gp.addMessage("Calculating final scores...")

gp.AddField(outFeatureClass,"TotalScore","DOUBLE")

factorScoreColumns $=$ factorScoreColumns[0:-3]

factorReclassColumns $=$ factorReclassColumns[0:-4]

gp.CalculateField(outFeatureClass,"TotalScore", factorScoreColumns)

gp.addMessage("Calculating unsuitable areas...")

gp.MakeFeatureLayer(outFeatureClass, "Restricted", factorReclassColumns)

gp.CalculateField("Restricted","TotalScore",0)

gp.addMessage("DONE!") 